\title{
Principal Fiber Bundle Interpretation of the KP-Hierarchy
}

\author{
By \\ Josef Dorfmeister* and Jacek SzMigielSKI*
}

\begin{abstract}
The Grassmannian and the Lax pair approaches to the Kadomtsev-Petviashvili (KP) hierarchy are analyzed in the framcwork of (formal) principal fiber bundles. The underlying factorization problcm is formulated as a local triviality condition. In particular the common object in the two approaches - the Baker function - coordinatizes the base space, the fiber consists of certain generalized differential operators.

An example is constructed to show that despite the apparent similarity to the splitting in [8], the local triviality condition cannot be given a group theoretic interpretation.
\end{abstract}

\section{Introduction}

For about twenty years "completely integrable" nonlinear partial differential equations have been investigated intensively from different points of view. In particular, the Kadomtsev-Petviashvili hierarchy (KP-hierarchy) has been treated from the point of view of Grassmannian like manifolds [12], [13], Lax pairs [6], [7], [13], [14], and the general Riemann-Hilbert problem [8]. The original goal of this paper was to clarify geometrically the relations between these three approaches to the KP-hierarchy. In the end we were able to generalize the pseudo-differential operator approach of [13] to give a natural relation between the Grassmannian point of view and the Lax pair point of view via (formal) principal fiber bundles. Even something like a Riemann-Hilbert splitting appears (Theorem 3.6 and (5.7.27)). However, it turns out that this Riemann-Hilbert type of splitting cannot be obtained as a "group splitting" as in [8]. At this point we have to leave it open to understand the relations between all these approaches from a higher, unifying approach.

In $\S 1$ we recall the definition of the Grassmannian manifold $X$ and the associated $\tau$-functions. In $\$ 2$ we consider a set $\mathscr{W}_{0}$ that will turn out in $\S 4$ to be a principal $G \ell\left(H_{+}\right)$-fiber bundle over $X$ and a map $\widehat{\varphi}$ from $\mathcal{W}_{0}$ to generalized pseudo-differential operators. In Theorem 2.6 we prove $\widehat{\varphi}_{W b}=\widehat{\varphi}_{b} \widehat{\varphi}_{W}, W \in \mathcal{W}_{0}$,

Communicated by M. Kashiwara, January 29. 1991.

1991 Mathematics Subject Classification: 58B25, 58F07, 35Q15.

* Department of Mathematics, University of Kansas, Lawrence, KS 66045, U.S.A. 
$b \in G \ell\left(H_{+}\right)$. This property of $\widehat{\varphi}$ will be crucial for the principal fiber bundle aspect of this paper.

In $\S 3$ we consider the usual flow $\Gamma_{+}$on the Grassmannian $X$ and introduce two generalized pseudo-differential operators $\widehat{\psi}_{W}^{+}$and $\widehat{\psi}_{W}^{-}, W \in \mathcal{W}_{0}$. Here $\widehat{\psi}_{W}^{-}$is the pseudo-differential operator associated with the Baker function $\psi_{W}$ in [13] and $\widehat{\psi}_{W}^{+}$is an infinite differential operator. One of the main results (Theorem 3.6) of this paper is the "splitting" $\widehat{\psi}_{W}^{+} \circ \widehat{\varphi}_{W}=\widehat{\psi}_{W}^{-}$. It allows us to define principal $G \ell\left(H_{+}\right)$fiber bundles of pseudo-differential operators in $\$ 4$. More precisely, since $X$ is a homogeneous space, $X=G / P$, we consider the principal $G \ell\left(H_{+}\right)$fiber bundle $E=G \underset{\mathrm{p}}{\times} G \ell\left(H_{+}\right)$. The main contents of $\S 4$ is to show that $E$ is isomorphic with $\mathcal{W}_{0}$ a as a principal fiber bundle and also with three (different but isomorphic) $G \ell\left(H_{+}\right)$-principal fiber bundles of generalized pseudo-differential operators. The base of these bundles (of course isomorphic with $X$ ) is given, roughly speaking, by $\left\{\widehat{\psi}_{W}^{-} ; W \in \mathcal{W}_{0}\right\}$ and the total space is given in one bundle as $\left\{\widehat{\varphi}_{W} ; W \in \mathcal{W}_{0}\right\}$, whereas it is given in another bundle as $\left\{\widehat{\psi}_{W}^{+} ; W \in \mathcal{W}_{0}\right\}$. This gives an interpretation of $\widehat{\psi}_{W}^{+}$and $\widehat{\psi}_{W}^{-}$as fiber and base elements in a principal fiber bundle.

Finally, we discuss differential equations associated with generalized Baker functions is Sections 3.8 and $\mathbf{3 . 9}$ and for $\widehat{\varphi}_{W}, \widehat{\psi}_{W}^{-}$in $\S 5$. The last section of this paper, 5.7, discusses the question of "invertibility" of $\widehat{\psi}^{+}$. This is of importance, since one would like to write $\widehat{\varphi}_{W}=\left(\widehat{\psi}_{W}^{+}\right)^{-1} \circ \psi_{W}^{-}$and to relate this splitting to the formally very similar splitting in [8]. We show that $\widehat{\psi}_{W}^{+}$is not invertible in any obvious sense, thus leaving the question open how this paper relates to [8].

\section{\$1. Notation and Basic Results}

1.1. We will use the notation of [2], but in this paper we will largely disregard Banach structures. For the convenience of the reader we recall the basic set-up.

Let $H=H_{+}+H_{-}$be the orthogonal sum of two infinite dimensional Hilbert spaces; e.g. $H=L^{2}\left(S^{1}\right), H_{+}=\operatorname{span}\left\{\lambda^{r} ; r \geq 0\right\}, H_{-}=\operatorname{span}\left\{\lambda^{r} ; r<0\right\}$. We choose subspaces $V^{+} \subset B\left(H_{+}, H_{-}\right), V^{-} \subset B\left(H_{-}, H_{+}\right)$such that $V=\left(V^{+}, V^{-}\right)$is a Jordan pair, i.e. for $x^{+} \in V^{+}, y^{-} \in V^{-}$, we have $x^{+} y^{-} x^{+} \in V^{+}$and $y^{-} x^{+} y^{-} \in$ $V^{-}$. In [2] we chose $V^{+}, V^{-}$as "symmetric normed ideal" and thus imposed canonical Banach structures on $V$; but for the purposes of this paper this seems to be only of minor importance. Nevertheless we have always these examples in mind.

1.2. Given $V=\left(V^{+}, V^{-}\right)$one constructs a variety $X$ by

$$
X=V^{+} \times V^{-} / \sim,
$$

where the equivalence relation " $\sim$ " is given by:

(1.2.2) $(x, y) \sim\left(x^{\prime}, y^{\prime}\right)$ if $I-\left(y-y^{\prime}\right) x$ is invertible and $x^{\prime}=x\left[I-\left(y-y^{\prime}\right) x\right]^{-1}$. 
The equivalence class containing $(x, y)$ will be denoted by $(x: y)$.

An interpretation of the points of $X$ as subspaces of $H$ is given as follows:

For $(x, y) \in V$ set

$$
W=\text { image }\left(\begin{array}{c}
I-y x \\
-x
\end{array}\right)
$$

Then the map $(x: y) \mapsto W$ is an isomorphism from $X$ onto some independently defined variety of closed subspaces of $H$ (see [2; Theorem 2.6]).

We also note that if $(x, y) \sim\left(x^{\prime}, y^{\prime}\right)$, then there exists some $g \in G \ell\left(H_{+}\right)$ such that

$$
\left(\begin{array}{c}
I-y x \\
-x
\end{array}\right)=\left(\begin{array}{c}
I-y^{\prime} x^{\prime} \\
-x^{\prime}
\end{array}\right) g
$$

Finally, we would like to recall that the sets $\left(V^{+}: y\right), y \in V^{-}$, form an atlas of $X$. Under suitable assumptions on the topology of $X$, these charts are all open and dense in $X$. Therefore, frequently only one chart is considered. We note that the chart $U_{0}=\left(V^{+}: 0\right)$ corresponds to those $(x, y)$, for which $I-y x$ is invertible. Large parts of this paper remain true for $V^{-}=B\left(H_{-}, H_{+}\right)$. However, for certain results it will be necessary to use $V^{-}=\left(V^{+}\right)^{+}=\left\{y \in B\left(H_{-}\right.\right.$, $\left.H_{+}\right), V^{+} y$ is of trace class $\}$.

1.3. The variety $X$ admits natural group actions. We have discussed this in [2] and found groups larger than the ones considered in [13] or [9]. Set

$$
G=\left\{\left(\begin{array}{ll}
a & b \\
c & d
\end{array}\right) \in G \ell(H) ; c \in V_{+}, a \text { is a Fredholm operator of index } 0\right\} .
$$

Then $G$ is connected and acts transitively on $X$. This action is described by the equation

$$
\left(\begin{array}{ll}
a & b \\
c & d
\end{array}\right) \quad\left(\begin{array}{c}
I-y x \\
-x
\end{array}\right)=\left(\begin{array}{c}
I-u v \\
-v
\end{array}\right) \mathrm{h}
$$

1.4. Of particular importance for differential equations related to $X$ is the subgroup $\Gamma_{+} \subset G$,

$$
\Gamma_{+}=\left\{M_{f} \in G ; f \in H^{\infty}\left(S^{1}\right), f(0)=1\right\}
$$

where $M_{f}$ denotes "multiplication by $f$ ".

Most of the time the $f$ 's under consideration are of the form $e^{t_{1} \lambda+t_{2} \lambda^{2}+\ldots}$, where the sum in the exponent is actually finite.

We note that $M_{f}$ is of the form

$$
M_{f}=\left(\begin{array}{ll}
a & b \\
0 & d
\end{array}\right)=\exp \left(t_{1} \Lambda+t_{2} \Lambda^{2}+\ldots\right)
$$


where $\Lambda=M_{\lambda}$ is the shift operator.

We recall from [13] that $b$ is of trace class if $f$ is twice differentiable. This applies of course, to the case where $f=e^{p}$ and $p$ is a polynomial in $\lambda$. In particular, all determinants and traces considered in this paper will trivially exist.

1.5. We recall some notation from $[2 ; \S \S 5,6]$. For $p \in X$ we set $\underline{t} \cdot p=\exp$ $\left(t_{1} \Lambda+t_{2} \Lambda^{2}+\ldots\right) \cdot p$, where the operation of $\Gamma_{+}$on $X$ is defined by (1.3.2) and (1.4.2).

Now let $x \in V^{+}$, then $(x: 0) \in X$ and also $\underline{t} \cdot(x: 0)=(x(\underline{t}): 0) \in X$ for $\underline{t}$ in a neighborhood of 0 . With the notation of (1.4.2) we know from $[2 ; 5.5 .1]$

$$
x(\underline{t})=d(\underline{t}) x(a(\underline{t})-b(\underline{t}) x)^{-1} .
$$

Moreover, [2;6] states

$$
\begin{aligned}
\partial_{h} x(\underline{t}) & =\Lambda_{-}^{k} x(\underline{t})-x(\underline{t}) \Lambda_{+}^{k}+x(\underline{t}) s_{k} x(\underline{t}), \\
x(\underline{t})_{i, J+1} & =x(\underline{t})_{t-1, J}-\partial_{1} x(\underline{t})_{t, J}+x(\underline{t})_{t, 0} x(\underline{t})_{-1, J}, \text { for } i<0 \text { and } j \geq 0 .
\end{aligned}
$$

Note that in (1.5.2) the matrices $\Lambda_{+}, s_{h}, \Lambda_{-}$are the natural nonzero components of the matrix $\Lambda$.

In $[2 ; 5.7]$ we considered $n_{\llcorner} \in \Gamma_{+}$, where

$$
n_{\zeta}(\lambda)=\exp \left(-\sum_{h=1}^{\infty} k^{-1} \zeta^{-h} \lambda^{k}\right)
$$

We set

$$
\begin{gathered}
\underline{n}_{\zeta}=-\left(k^{-1} \zeta^{-k}\right)_{k=1}^{x}, \\
m(\underline{t} ; \zeta)=1+\sum_{j=0}^{x} x(\underline{t})_{-l j} \zeta^{-(\jmath+1)} \\
\tau(\underline{t} ; x)=\operatorname{det}\left(I-b(\underline{t}) \times a(\underline{t})^{-1}\right) .
\end{gathered}
$$

Then from [2; Lemma 5.7] we know

$$
m(\underline{t}, \zeta)=\frac{\tau(\underline{t}+\underline{n} \xi ; x)}{\tau(\underline{t} ; x)}
$$

Note that $m$ is essentially the first row of $x(t)$. Moreover, [2; 5.8.2] shows

$$
\partial_{1} \ln \tau(\underline{t} ; x)=-x(\underline{t})_{-1,0}
$$

Finally, we recall the Baker function

$$
\psi(\underline{t} ; \xi)=\exp \left(\sum_{k=1}^{\infty} \xi^{k} t_{k}\right) m(\underline{t} ; \zeta)
$$

\section{\$2. A Map into Generalized Pseudo-Differential Operators}

2.1. Let $a(x, \cdot) \in L^{2}\left(S^{1}\right)$ for $x \in C$ and $A_{\kappa}(x)$ be the $\kappa$-th Fourier coefficient of 
$a(x, \cdot)$. We assume that $A_{\kappa}(x)$ is $C^{x}$ for each $\kappa \in Z$.

Definition 1. A generalized pseudo-differential operator $A\left(x, \partial_{\varkappa}\right)$ with symbol $\sigma(A)=a \in L^{2}\left(S^{1}\right)$ is a formal expression

$$
A\left(x, \partial_{\imath}\right)=\sum_{\kappa \in Z} A_{K}(x) \partial_{r}^{K}
$$

We will frequently use the following convention

$$
a(x, \lambda) e^{\lambda x}=A\left(x, \partial_{x}\right) e^{\lambda x}
$$

in which

$$
\partial_{r}^{\kappa} e^{\lambda x}=\partial^{\kappa} e^{\lambda x}, \text { for } \kappa \in Z
$$

We denote the vector space of generalized pseudo-differential operators by $\Psi$. $\Psi$ is not an algebra and in fact this is not required in our context. We will introduce a smaller subset of $\Psi$ (see Section 4.3) which will have the structure of a $G L\left(H_{+}\right)$module. There, we adopt the standard rules of composing pseudodifferential operators. They are determined by the generalized Leibniz rule:

$$
\begin{aligned}
\partial_{x}^{K} f & =\sum_{J=0}^{K}\left(\begin{array}{c}
\kappa \\
j
\end{array}\right) \frac{\partial^{J} f}{\partial x^{j}} \partial_{x}^{\kappa-J}, \quad \kappa \geq 0, \\
\partial_{x}^{-\kappa} f & =\sum_{J=0}^{\infty}(-1)^{\prime}\left(\begin{array}{c}
\kappa+j-1 \\
j
\end{array}\right) \frac{\partial^{J} f}{\partial x^{\prime}} \partial_{\imath}^{-(\kappa+I)}, \quad \kappa \geq 0 .
\end{aligned}
$$

Let

$$
\mathscr{W}=\left\{\left(\begin{array}{c}
w_{+} \\
w_{-}
\end{array}\right) ; w_{+} \in B\left(H_{+}\right), w_{-} \in V^{+}\right\}
$$

We set

$$
W^{*}=\left\{w \in W ; w_{+} \in G L\left(H_{+}\right)\right\} .
$$

We also write $\boldsymbol{l}=\lambda^{0}$ and subsequently compute the column with index "O" of some element $W \in \mathcal{W}$ by evaluating $W \boldsymbol{l}$.

In Section 3 we will be interested in pseudo-differential operators which are defined on $\Gamma_{+}$, i.e. all $A_{\kappa}$ 's in Definition 1 are functions $\Gamma_{+} \rightarrow C$. We recall that $e^{\mathrm{x} \lambda} \in \Gamma_{+}$for any $x \in C$. Moreover, a general element $g \in \Gamma_{+}$can by parametrized as $g\left(x, t_{2}, t_{3}, \ldots\right)=e^{x \lambda+t_{2} \lambda^{2}+t_{3} \lambda^{2}+\ldots}$ with $x, t_{2}, t_{3}, \ldots \in C$. We will frequently write $g=e^{x \lambda+q(\lambda)}=e^{q(x, \lambda)}$.

Definition 2. For $g \in \Gamma_{+}$we define $\widehat{\varphi}: \mathcal{W} \rightarrow \Psi$, by

$$
\sigma\left(\widehat{\varphi}_{W}(g)\right)=\left(g^{-1} W\right) \boldsymbol{l},
$$

where $g^{-1} W=M_{g}^{-1} W T_{g}$. Here $M_{g}$ denotes the multiplication operator by 
$g, T_{g}=\Pi_{+} M_{g} \Pi_{+}$and $\Pi_{+}$is the canonical orthogonal projection on $H_{+}$.

Remark. (1) Since in Definition 2 we have $g \in \Gamma_{+} \subset H_{+} \cap H^{\varkappa}$, and since $W T_{g} \mathbb{I}=W g$ holds; the condition (2.1.5) means

$$
\sigma\left(\widehat{\varphi}_{W}(g)\right)=\left(g^{-1} W\right) \dot{g} .
$$

In what follows we will use the same symbol to denote both an element $g \in \Gamma_{+}$ as well as the corresponding multiplication operator $M_{g}$.

(2) We will frequently use another way of defining $\widehat{\varphi}$. We set

$$
\begin{gathered}
\varphi: \mathscr{W} \times \Gamma_{+} \mapsto L^{2}\left(S^{1}\right), \\
\varphi_{W}(g)=\widehat{\varphi}_{W}(g) g=g \sigma\left(\widehat{\varphi}_{W}(g)\right)=\sigma\left(\widehat{\varphi}_{W}(g)\right) g .
\end{gathered}
$$

In (2.1.7) we use the explicit parametrization of $g$ in terms of $x, t_{2}, t_{3}, \ldots$ and the rule

$$
\partial_{x}^{\kappa} e^{x \lambda+q(\lambda)}=\lambda^{\kappa} e^{x \lambda+q(\lambda)} .
$$

In view of (2.1.6) and (2.1.7) we have

$$
\varphi_{W}(g)=W g \text {. }
$$

From now on a Greek letter with the hat " $\wedge$ " means an element of $\Psi$, i.e. $\widehat{\varphi}$, $\widehat{\chi}, \in \Psi$ etc., on the other hand, $\varphi, \chi$, etc. (without " $\wedge$ ") denote the functions in $L^{2}\left(S^{1}\right)$ obtained from $\widehat{\varphi}$ and $\widehat{\chi}$ via $(2.1 .8)$.

In the next few sections we will study the dependence of the coefficients of $\widehat{\varphi}_{W}(g)$ on $x$. In this case we will frequently write $\widehat{\varphi}_{W}\left(e^{q(x)}\right)$ allowing $x$ to vary over $C$, while keeping $q(\lambda)$ fixed. We also note that (2.1.5) can be written as

$$
\sigma\left(\widehat{\varphi}_{W}(g)\right)=\left(\begin{array}{ll}
a & b \\
0 & d
\end{array}\right)\left(\begin{array}{l}
w_{+} \\
w_{-}
\end{array}\right) a^{-1} \mathbb{1}
$$

where $a, b, d$ denote the matrix blocks of $g^{-1}$ and $W=\left(\begin{array}{l}w_{+} \\ w_{-}\end{array}\right)$.

It is easy to see that $\sigma\left(\widehat{\varphi}_{W}\left(e^{q(x)}\right)\right) \in L^{2}\left(S^{1}\right)$ for each $x \in \mathbb{C}$. Moreover, in Corollary 2.2.1 we will show that $\sigma\left(\widehat{\varphi}_{W}\left(e^{q(x)}\right)\right)$ is an entire function of $x$. These two facts ensure that $\widehat{\varphi}_{W}$ is a well defined map from $\mathcal{W}$ into $\Psi$.

2.2. In this section we continue to study $\varphi$. First we recall that a basis $\left\{\psi_{1}\right\}$ of the Hilbert space $H$ that is obtained from an orthonormal basis by a bounded invertible operator is called a basis equivalent to an orthonormal basis [3].

Lemma. Let $n \in \mathbb{Z}, g \in \Gamma_{+}$. Then the sequence $\left\{u_{n}\right\}$, where $u_{n}(g)=\lambda^{n} g$, $n \in Z$, is a basis of $L^{2}\left(S^{1}\right)$ equivalent to the canonical basis $\left\{\lambda^{n}\right\}$. Moreover, the sequence $\left\{u_{n}\right\}_{n=0}^{\infty}$ is a basis of $H_{+}$equivalent to $\left\{\lambda^{n}\right\}_{n=0}^{\infty}$. 
Proof. It suffices to notice that $u_{n}(g)=M_{g} \lambda^{n}$, where $M_{g}$ is an invertible bounded operator on $L^{2}\left(S^{1}\right)$, given by $M_{g} f=g f$.

2.3. We recall that any $g \in \Gamma_{+}$can be written as $g(\lambda)=e^{x \lambda+q(\lambda)}$, where $q(\lambda)$ is holomorphic in $\boldsymbol{D}=\{\lambda \in \boldsymbol{C},|\lambda|<1\}, q(0)=0, q^{\prime}(0)=0$, and $x \in \boldsymbol{C}$. Let us fix $q$ and allow $x$ to vary over $C$. We set $q(x, \lambda)=x \lambda+q(\lambda)$. Next we define the maps

$$
u_{n}^{q}: C \rightarrow L^{2}\left(S^{1}\right), \quad x \rightarrow u_{n}\left(e^{q(x, \lambda)}\right)
$$

We note that the functions $u_{n}^{q}(x)$ are entire. This follows from $u_{n}^{q}(x)=$ $g_{0}(\lambda) \sum_{\kappa=0}^{\infty} \frac{\lambda^{k+n} x^{\kappa}}{k !}, g_{0}(\lambda)=e^{q(\lambda)}$, since

$$
\left\|g_{0}(\lambda) \frac{\lambda^{\kappa+n}}{\kappa !} x^{\kappa}\right\|_{2} \leq C \frac{|x|^{\kappa}}{\kappa !} \text { for some } C>0
$$

In addition to this we will need

Lemma. Let $h: C \mapsto L^{2}\left(S^{1}\right), x \rightarrow h(x)$ be an entire map. Then $h(x)=$ $\sum_{n \in Z} h_{n}(x) u_{n}^{q}(x)$ where $h_{n}: C \mapsto C$ is entire.

Proof. Set $v_{m}^{q}(x)=\lambda^{m} e^{-q \overline{(x, \lambda)}}$, then $\left\langle u_{n}^{q}(x), v_{m}^{q}(x)\right\rangle=\delta_{n, m}$. Hence $h_{n}(x)=$ $\left\langle h(x), \quad v_{n}^{q}(x)\right\rangle=\left\langle e^{-q(x, \lambda)} h(x), \lambda^{n}\right\rangle$. Since here the first vector is entire, $e^{-q(x, \lambda)} h(x)$ is $C^{1}$ on any open subset of $C$. Since the scalar product is continuous we get that $h_{n}(x)$ is $C^{1}$ on any open subset of $C$, thus showing that $h_{n}(x)$ is entire.

2.4. We will apply the above results to the symbol of $\widehat{\varphi}_{W}(g)$.

Lemma. Let $W \in \mathcal{W}$, then

$$
W u_{0}(g)=g \sigma\left(\widehat{\varphi}_{W}(g)\right)=\varphi_{W}(g)
$$

As a consequence, the coefficients of $\lambda^{m}$ in $\sigma\left(\widehat{\varphi}_{W}(g)\right)$ are the same as the coefficients of $W u_{0}(g)=\varphi_{W}(g)$ relative to the basis $\left\{u_{n}(g)\right\}$.

Proof. The claim follows directly from (2.1.7) and (2.1.8).

As to the $x$-dependence of $\sigma\left(\widehat{\varphi}_{W}(g)\right)$ we have the following

Corollary 1. Let $q(x, \lambda)=x \lambda+q(\lambda)$. Then for a fixed $q(\lambda)$ and any $W \in W$ the map $x \mapsto \sigma\left(\widehat{\varphi}_{W}\left(e^{q(x, \lambda)}\right)\right)$ is an entire map from $C$ to $L^{2}\left(S^{1}\right)$.

Corollary 2. $\widehat{\varphi}_{W}\left(e^{q(x, \lambda)}\right) \in \Psi$ and all the Fourier coefficients of $\sigma\left(\widehat{\varphi}_{W}\left(e^{q(x)}\right)\right)$ are entire functions of $x$. 
2.5. Above we have described $W u_{0}\left(e^{q(x, \lambda)}\right)$. Next we consider $W u_{\kappa}\left(e^{q(x, \lambda)}\right)$.

Lemma. Let $W=\mathcal{W}$, then $W u_{\kappa}\left(e^{q(x, \lambda)}\right)=\partial_{x}^{k} W u_{0}\left(e^{q(x, \lambda)}\right)$, for $\kappa \geq 0$. In particular, relative to the basis $\left\{u_{m}\left(e^{q(x, \lambda)}\right)\right\}$ of $H$ and $\left\{u_{l}\left(e^{q(x)}\right)\right\}_{l=0}^{\infty}$ of $H_{+}$, the matrix entries of $W$ satisfy

$$
W_{m, K}(x)=\sum_{\iota=0}^{k}\left(\begin{array}{c}
\kappa \\
\iota
\end{array}\right) W_{\mathrm{m}-(\kappa-\imath), 0}^{(1)}(x)
$$

Proof. The map $x \rightarrow W u_{K}\left(e^{q(x, \lambda)}\right)$ is entire. Since $u_{K}\left(e^{q(x, \lambda)}\right)=\partial_{x}^{K} u_{0}\left(e^{q(x, \lambda)}\right)$ we have $W u_{\kappa}\left(e^{q(x, \lambda)}\right)=W \partial_{x}^{K} u_{0}\left(e^{q(x, \lambda)}\right)=\partial_{x}^{K} W u_{0}\left(e^{q(x, \lambda)}\right)=\partial_{x}^{K} \sum_{r \in Z} W_{r, 0}(x) u_{r}\left(e^{q(x, \lambda)}\right)$ $=\sum_{r \in \boldsymbol{Z}} \sum_{\iota=0}^{\kappa}\left(\begin{array}{l}\kappa \\ \iota\end{array}\right) W_{r, 0}^{(\iota)}(x) u_{r+\kappa-\iota}\left(e^{q(x, \lambda)}\right)=\sum_{m \in \boldsymbol{Z}}\left[\sum_{\iota=0}^{K}\left(\begin{array}{l}\kappa \\ \iota\end{array}\right) W_{m-(\kappa-\iota), 0}^{(\iota)}(x)\right] u_{m}\left(e^{q(x, \lambda)}\right)$.

Since $W u_{\kappa}\left(e^{q(x, \lambda)}\right)=\sum_{\iota=0}^{\kappa} W_{m, \kappa}(x) u_{m}\left(e^{q(x, \lambda)}\right)$, the claim follows.

The claim of the lemma holds pointwise in $x$, thus the lemma holds for any $g \in \Gamma_{+}$if interpreted properly. To see this we start with a given $g=e^{x_{1} \lambda+q(\lambda)}$, then we extend it to $e^{x \lambda+q(\lambda)}$, apply the lemma and finally evaluate all the statements in the lemma at $x=x_{0}$. $W=W^{\prime}$.

Corollary. Let $W$ and $W^{\prime} \in \mathcal{W}$ and $\sigma\left(\widehat{\varphi}_{W}\left(e^{q(x, \lambda)}\right)=\sigma\left(\widehat{\varphi}_{W^{\prime}}\left(e^{q(x, \lambda)}\right)\right.\right.$, then

Proof. From Lemma 2.4, it follows that $W u\left(e^{q(x, \lambda)}\right)=W^{\prime} u\left(e^{q(1, \lambda)}\right)$. Thus from the lemma above we obtain $W u_{\kappa}\left(e^{q(x, \lambda)}\right)=W^{\prime} u_{\kappa}\left(e^{q(x, \lambda)}\right)$ for all $\kappa \geq 0$, which proves the claim.

2.6. The following result is fundamental for this paper.

Theorem. The map $\widehat{\varphi}: \mathscr{W} \mapsto \Psi$ is injective and $\widehat{\varphi}_{W b}=\widehat{\varphi}_{b} \widehat{\varphi}_{W}$ for all $b \in$ $B\left(H_{+}\right) \subset \mathcal{W}, W \in \mathcal{W}$. In particular, setting $\psi_{+}^{+}=\widehat{\varphi}\left(G L\left(H_{+}\right)\right), G L\left(H_{+}\right)$is (anti)isomorphic to $\psi_{+}^{+}$.

Proof. We have already proved injectivity in Corollary 2.5. To prove $\widehat{\varphi}_{W b}=\widehat{\varphi}_{b} \widehat{\varphi}_{W}$ we note (see 2.1.7)

$$
\widehat{\varphi}_{W b}(g) g=\varphi_{W b}(g)=W b g \quad \text { for } g \in \Gamma_{+} .
$$

Let $g=e^{q(x, \lambda)}$, then

$$
\begin{gathered}
\varphi_{W b}\left(e^{q(x, \lambda)}\right)=W b e^{q(x, \lambda)}=W b u_{0}\left(e^{q(x, \lambda)}\right)=W \sum_{m \geq 0} b_{m, 0}(x) u_{m}\left(e^{q(x, \lambda)}\right) \\
=\sum_{m \geq 0} b_{m, 0}(x) W u_{m}\left(e^{q(x, \lambda)}\right)=\sum_{m \geq 0} b_{m, 0}(x) \partial_{x}^{m} W u_{0}\left(e^{q(x, \lambda)}\right)
\end{gathered}
$$


where in the last step we used Lemma 2.5. We see now that by Lemma 2.4 and $(2.1 .7)$

$$
\sum_{m \geq 0} b_{m, 0}(x) \partial_{\imath}^{m}=\widehat{\varphi}_{b}\left(e^{q(x, \lambda)}\right) .
$$

Similarly by Lemma $2.4, W u_{0}\left(e^{q(x, \lambda)}\right)=\varphi_{W}\left(e^{q(x, \lambda)}\right)$. We thus have

$$
\widehat{\varphi}_{W b}\left(e^{q(x, \lambda)}\right) e^{q(x, \lambda)}=\widehat{\varphi}_{b}\left(e^{q(x, \lambda)}\right) \varphi_{W}\left(e^{q(x, \lambda)}\right)=\widehat{\varphi}_{b}\left(e^{q(x, \lambda)}\right) \widehat{\varphi}_{W}\left(e^{q(x, \lambda)}\right) e^{q(x, \lambda)},
$$

which completes the proof.

\section{§3. "Factorization" of $\widehat{\varphi}_{W}(g)$ and Generalized Baker Functions}

3.1. We will be interested in the subset $\mathcal{W}_{0} \subset \mathcal{W}$ defined by

$$
\begin{aligned}
\mathcal{W}_{0}= & \left\{W=\left(\begin{array}{l}
w_{+} \\
w_{-}
\end{array}\right) \in W: W: H_{+} \rightarrow W\left(H_{+}\right)\right. \text {is injective and } \\
& \left.w_{+} \text {is a Fredholm operator of index } 0\right\}
\end{aligned}
$$

Let us denote by $p(W) \subset L^{2}\left(S^{1}\right)=H$ the image of $H_{+}$under $W$. We have the following result

Lemma. a) $p(W)$ is a closed subspace of $H$.

b) $W: H_{+} \rightarrow p(W)$ is a bounded isomorphism of vector spaces.

Proof. Clearly, $W: H_{+} \rightarrow p(W)$ is an isomorphism of vector spaces. Also, this map is bounded. We claim that $T=W^{-1}$ is bounded. This is equivalent with

(*) There exists some $\varepsilon>0$ such that for all $x \in H_{+}$with $\|x\|=1: \varepsilon<\|W x\|$.

Suppose this is not true, then for $\varepsilon=\frac{1}{n}$ there exists some $x_{n} \in H_{+}$such that $\left\|x_{n}\right\|=1$ and $\left\|W x_{n}\right\| \leq \frac{1}{n}$. Since $w_{+}$is Fredholm of index 0 we know $w_{+}=a_{+}+k_{+}$, where $a_{+} \in G \ell\left(H_{+}\right)$and $k_{+}$is compact. Since the sequence $\left(x_{n}\right)$ is bounded, $\left(k_{+} x_{n}\right)$ contains a convergent subsequence. Choosing a subsequence of $\left(x_{n}\right)$ we can assume that $\left(k_{+} x_{n}\right)$ converges. Repeating this argument we can also assume that $\left(w_{-} x_{n}\right)$ converges. From $\left\|W x_{n}\right\| \leq \frac{1}{n}$ we obtain $w_{-} x_{n} \rightarrow 0$ and $a_{+} x_{n}+k_{+} x_{n} \rightarrow 0$. Therefore $\left(x_{n}\right)$ converges in $H_{+}$, say to $x$. But then $\|x\|=\lim _{n}\left\|x_{n}\right\|=1$ and $\|W x\|=\lim _{n}\left\|W_{n}\right\|=0$, whence $x \in \operatorname{Ker} W$. This yields a contradiction, since Ker $W=0$ by assumption. As a consequence, $p(W)$ is a complete subspace of $H$, thus closed.

3.2. The relation between $\mathcal{W}_{0}$ and $X$ (see (1.2.1)) is given by the following lemma. 
Lemma. The map $p: \mathscr{W}_{0} \rightarrow X, W \rightarrow p(W)$ is onto.

Proof. This is plain from the fact that in view of Lemma 3.1 we have $p\left(\mathcal{W}_{0}\right)=G r(H)$, where $\operatorname{Gr}(H)$ is defined in [13; Theorem 2.6], see also [2; 2.2]. Moreover, [2; Theorem 2.6] shows that $G r(H)$ is isomorphic to $X$.

3.3. There is a natural right action of $G \ell\left(H_{+}\right)$on $\mathcal{W}_{0}$. More specifically, for $g \in G L\left(H_{+}\right)$, we set

$$
W g=\left(\begin{array}{l}
w_{+} g \\
w_{-} g
\end{array}\right)
$$

We recall that a group $G$ acts freely on a set $B$ if $g b=b$ for some $g \in G$ and $b \in B$ implies $g=e$, where $e$ is the identity element of $G$.

Lemma. $G L\left(H_{+}\right)$acts freely on $\mathcal{W}_{0}$.

Proof. $W g=W$ for some $W \in W_{0}$ and $g \in G L\left(H_{+}\right)$implies $W(e-g)=0$. Hence also for every $a \in H_{+}$we have $W(e-g) a=0$. Thus $(e-g) a \in \operatorname{Ker} W$ for all $a \in H_{+}$. By Lemma 3.1 however, $\operatorname{Ker} W=0$, thus $(e-g) a=0$ for all $a \in H_{+}$, whence $e=g$.

3.4. From now on we assume $V^{-}=\left(V^{+}\right)^{*}=\left\{y \in B\left(H_{-}, H_{+}\right), V^{+} y \subset B_{1}\left(H_{-}\right.\right.$, $\left.\left.H_{+}\right)\right\}$, where $B_{1}\left(H_{-}, H_{+}\right)$denotes the trace class operators.

Following [13] we introduce for $W \in X$ the set

$$
\Gamma_{+}^{W}=\left\{g \in \Gamma_{+} ; g^{-1} W \in U_{0}\right\} .
$$

We recall from 1.2 that $U_{0}=\left\{(x: 0), x \in V^{+}\right\}$is an open and dense set in $X$.

In what follows we give $\Gamma_{+}$the topology induced from the inclusion $\Gamma_{+} \subset G$, where $G$ is defined in (1.3.1).

Lemma 1. $\Gamma_{+}^{W}$ is open and dense in $\Gamma_{+}$.

Proof. Let $W=(x: y)$ and $g^{-1}=\left(\begin{array}{ll}a & b \\ 0 & d\end{array}\right) \in \Gamma_{+}$, then we have

$$
\Gamma_{+}^{W}=\left\{g \in \Gamma_{+} ; I-a y x a^{-1}-b x a^{-1} \in G \ell\left(H_{+}\right)\right\} .
$$

It is not hard to verify that the map $P \rightarrow B\left(H_{+}\right), g \mapsto I-a y x a^{-1}-b x a^{-1}$ is continuous, where $P$ denotes the stabilizer of $H_{+}$in $G$. Hence $\Gamma_{+}^{W}$ is open in $\Gamma_{+}$. On the other hand, by [2; Theorem 2.5.1] we can choose $W=(x: y)$ so that $y$ is of finite rank. Since also $b$ is of trace class, we see that $\varphi(g)=\operatorname{det}\left(I-a y x a^{-1}-\right.$ $b x a^{-1}$ ) exists and is continuous for all $g \in P$. Finally, using [11, Theorem 3.5(b)] we obtain $\varphi(g) \neq 0$ if and only if $I-a y x a^{-1}-b x a^{-1} \in G \ell\left(H_{+}\right)$. Thus 


$$
\Gamma_{+}^{W}=\left\{g \in \Gamma_{+} ; \operatorname{det}\left(I-a y x a^{-1}-b x a^{-1}\right) \neq 0\right\} .
$$

In view of [13; Proposition 8.6] and Lemma 2 below we see that $\Gamma_{+}^{W}$ is dense in $\Gamma_{+}$. simple

We would like to remark that $\Gamma_{+}^{H_{+}}=\Gamma_{+}$. Moreover, we have the following

Lemma 2. Let $h \in \Gamma_{+}$. Then $\Gamma_{+}^{h W}=h^{-1} \Gamma_{+}^{W}$ for any $W \in X$.

Since it is rather easy to give examples of $W^{\prime} \mathrm{s}$ in $X$ for which $\Gamma_{+}^{W} \neq \Gamma_{+}$, we know that the sets $\Gamma_{+}^{W}$ are not trivially identical. Their role in our framework will become clear in the following section.

Finally we remark that $\Gamma_{+}$acts on $\mathcal{W}_{0}$ by $g \cdot W=g W a^{-1}$. For $W \in \mathcal{W}_{0}$ we set

$$
\widetilde{\Gamma}_{+}^{W}=\left\{g \in \Gamma_{+} ; g^{-1} \cdot W \in W^{*}\right\} .
$$

For $h \in G \ell\left(H_{+}\right)$it is straightforward to check

$$
\widetilde{\Gamma}_{+}^{W}=\widetilde{\Gamma}_{+}^{W h}
$$

This implies that $\widetilde{\Gamma}_{+}^{W}$ actually only depends on the image $p(W)$ of $W$ in $X$. Moreover,

$$
\widetilde{\Gamma}_{+}^{W}=\Gamma_{+}^{p(W)} .
$$

Therefore we will not distinguish between $\widetilde{\Gamma}_{+}^{W}$ and $\Gamma_{+}^{p(W)}, W \in \mathcal{W}_{0}$.

3.5. In this section we will use the construction outlined in $\mathbf{1 . 2}$ to write a given $\widehat{\varphi}_{W}, W \in W_{0}$, as a product of generalized pseudo-differential operators. From (2.1.7) we know that for $W \in \mathscr{W}$ and $g \in \Gamma_{+}$we have

$$
\widehat{\varphi}_{W}(g) g=g \sigma\left(\widehat{\varphi}_{W}(g)\right)
$$

where, by (2.1.5),

$$
\sigma\left(\widehat{\varphi}_{W}(g)\right)=g^{-1} . W 1 .
$$

The matrix form of $g^{-1} \cdot W$ is

$$
g^{-1} \cdot W=\left(\begin{array}{ll}
a & b \\
0 & d
\end{array}\right)\left(\begin{array}{l}
W_{+} \\
W_{-}
\end{array}\right) a^{-1}=\left(\begin{array}{c}
a w_{+} a^{-1}+b w_{-} a^{-1} \\
d w_{-} a^{-1}
\end{array}\right) .
$$

We restrict (3.5.1) to $g \in \Gamma_{+}^{W}$, thus ensuring that

$$
Q(g)=a w_{+} a^{-1}+b w_{-} a^{-1}
$$

belongs to $G L\left(H_{+}\right)$. Therefore, whenever $g \in \Gamma_{+}^{W}$, we can factor $g^{-1} \cdot W$ as

$$
g^{-1} \cdot W=\left(\begin{array}{c}
I \\
w(g)
\end{array}\right) Q(g) .
$$


We note that for $w_{+}=I$ and $w_{-}=-x$ we have $w(g)=-x(-\underline{t})$ as defined in (1.5.1).

To obtain the symbol $\sigma\left(\widehat{\varphi}_{W}(g)\right)$ one applies $g^{-1} \cdot W$ to $\mathbb{1}$. This suggests factoring $\widehat{\varphi}_{W}(g)$ according to (3.5.3). First, we define a generalized differential operator $\widehat{\psi}_{W}^{+}(g)$ by

$$
\sigma\left(\widehat{\psi}_{W}^{+}(g)\right)=Q^{-1}(g) \mathbb{1}, \quad g \in \Gamma_{+}^{W}
$$

Furthermore, we define a monic pseudo-differential operator $\widehat{\psi}_{W}^{-}(g)$ by

$$
\sigma\left(\widehat{\psi}_{W}^{-}(g)\right)=\left(\begin{array}{c}
I \\
w(g)
\end{array}\right), \quad g \in \Gamma_{+}^{W} .
$$

We note that

$$
g \sigma\left(\widehat{\psi}_{W}^{-}(g)\right)=\psi_{W}^{-}(g)
$$

is usually called the "Baker function" associated with $W \in X$.

We introduce some more notation. We denote by $\mathscr{P}_{+}\left(\Gamma_{+}^{W}\right)$, respectively $\mathscr{P}_{-}\left(\Gamma_{+}^{W}\right)$ the generalized pseudo-differential operators for which only nonnegative (resp. nonpositive) powers of $\partial_{x}$ occur. We assume further that the coefficients of these operators are defined only on $\Gamma_{+}^{W}$. For the sake of comparison, let us recall that $\widehat{\varphi}_{W}$ was defined on the entire $\Gamma_{+}$. Hereafter we will frequently use the restriction of $\widehat{\varphi}_{W}$ to $\Gamma_{+}^{W}$ denoted by $\widehat{\varphi}_{W \mid \Gamma_{+}^{W}}$. We clearly have $\widehat{\psi}_{W}^{ \pm} \in \mathscr{P}_{ \pm}\left(\Gamma_{+}^{W}\right)$.

3.6. The following theorem expresses $\widehat{\varphi}_{W}$ in terms of $\widehat{\psi}_{W}^{+}(g)$ and $\widehat{\psi}_{W}^{-}(g)$. This result is a geometric analogue of the lower and upper Volterra operators introduced in the context of direct scattering on $\mathbb{R}$ for the KP equation by [15]. An algebraic version of these operators appears in [8].

Theorem. (a) $\widehat{\psi}_{W}^{+}(g) \circ \widehat{\varphi}_{W}(g)=\widehat{\psi}_{W}^{-}(g), g \in \Gamma_{+}^{W}$. Moreover, if $p_{+} \in \mathscr{P}_{+}\left(\Gamma_{+}^{W}\right)$ and $p_{+}(g) \circ \widehat{\varphi}_{W}(g)=p_{-}(g)$ for all $g \in \Gamma_{+}^{W}$, then $p_{+}(g)=\widehat{\psi}_{W}^{+}(g)$ and $p_{-}(g)=\widehat{\psi}_{W}^{-}(g)$ for all $g \in \Gamma_{+}^{W}$.

(b) For each $W \in \mathcal{W}_{0}$ and each $g \in \Gamma_{+}^{W}$ there is a unique element $\chi_{W}^{-}(g) \in p(W) \in$ $X$ and a unique element $\chi_{W}^{+}(g) \in H_{+}$, such that

$$
\begin{aligned}
& \chi_{W}^{-}(g)=g\left(1+\sum_{n=1}^{\infty} a_{n}(g) \lambda^{-n}\right), \\
& \chi_{W}^{-}(g)=W \chi_{W}^{+}(g) .
\end{aligned}
$$

(c) Let $W \in W_{0}$. Then for the functions $\chi_{W}^{+}$and $\chi_{W}^{-}$associated to $W$ in (b) we have

$$
\begin{aligned}
& \chi_{W}^{-}(g)=g \sigma\left(\widehat{\psi}_{W}^{-}(g)\right), \\
& \chi_{W}^{+}(g)=g \sigma\left(\widehat{\psi}_{W}^{+}(g)\right) .
\end{aligned}
$$

(d) Let $W \in \mathcal{W}_{0}$ and choose $\chi_{W}^{+}(g)$ as in (b). Define $\widehat{\psi}_{W}^{-}(g)$ by (3.6.3) and $\widehat{\psi}_{W}^{+}(g)$ 
by (3.6.3). Then $\widehat{\psi}_{W}^{+}(g) \circ \widehat{\varphi}_{W}(g)=\widehat{\psi}_{W}^{-}(g)$.

Proof. Part (b) follows from [13; Proposition 5.1] and from the fact that for $W \in W_{0}$ the map $W: H_{+} \rightarrow p(W)$ is bijective. To prove (a) we start from the defining equation (3.5.3)

$$
g^{-1} \cdot W=\left(\begin{array}{c}
I \\
w(g)
\end{array}\right) Q(g)
$$

which via (3.5.4) and (3.5.5) determines $\widehat{\psi}_{W}^{+}$and $\widehat{\psi}_{W}^{-}$. We rewrite (1) using (3.5.1) to obtain

$$
\left(\begin{array}{c}
w_{+} \\
w_{-}
\end{array}\right) a^{-1} Q^{-1}(g)=M_{g}\left(\begin{array}{c}
I \\
w(g)
\end{array}\right)
$$

Now we apply both sides to the constant function 1 and make use of the definitions of $\widehat{\psi}_{W}^{+}$and $\widehat{\psi}_{W}^{-}$. Thus we obtain (interpreting $\sigma\left(\widehat{\psi}_{W}^{+}\right)$and $g \sigma\left(\widehat{\psi}_{W}^{+}\right)$as elements of $H_{+}$)

$$
\left(\begin{array}{l}
w_{+} \\
w_{-}
\end{array}\right) g \sigma\left(\widehat{\psi}_{W}^{+}(g)\right)=g \sigma\left(\widehat{\psi}_{W}^{-}(g)\right) .
$$

Applying the remark after (2.1.7) we have $g \sigma\left(\widehat{\psi}_{W}^{+}(g)\right)=\widehat{\varphi}_{W}^{+}(g) g$. Therefore the lefthand side of (3) can be rewritten as

$$
\left(\begin{array}{l}
w_{+} \\
w_{-}
\end{array}\right) \widehat{\psi}_{W}^{+}(g) g=\widehat{\psi}_{W}^{+}(g)\left(\begin{array}{l}
w_{+} \\
w_{-}
\end{array}\right) g .
$$

But the last term is just $\left(\widehat{\psi}_{W}^{+}(g) \circ \widehat{\varphi}_{W}(g) g\right.$ where "o" denotes the composition of pseudo-differential operators and where we used (2.1.7) and (2.1.8). Hence, altogether we obtain

$$
\widehat{\psi}_{W}^{-}(g)=\widehat{\psi}_{W}^{+}(g) \circ \widehat{\varphi}_{W}(g), \quad \text { for } g \in \Gamma_{+}^{W} .
$$

The uniqueness of (5) follows from the fact that (5) used along with (2.1.7) and (2.1.8) implies (3), which by (b) shows that (5) is unique. This finishes the proof of (a). To show (c) we start with $\widehat{\psi}_{W}^{ \pm} \in \mathscr{P}_{ \pm}\left(\Gamma_{+}^{W}\right)$ for which (a) is true, whence (5) is true. But (5) implies (3) and thus, if we define $\chi_{W}^{-}$and $\chi_{W}^{+}$by (6) and (7), we obtain (b). To verify (d) we define $\widehat{\psi}_{W}^{-}(g)$ and $\widehat{\psi}_{W}^{+}(g)$ by

$$
\begin{aligned}
& \chi_{W}^{-}(g)=g \sigma\left(\widehat{\psi}_{W}^{-}(g)\right), \\
& \chi_{W}^{+}(g)=g \sigma\left(\widehat{\psi}_{W}^{+}(g)\right) .
\end{aligned}
$$

From (3.6.2) we get (3) which implies (5) and thus.we have that (a) holds.

3.7. This section contains material parallel to that of $\mathbf{2 . 2}$. First we will slightly generalize the results from the last section. Let 


$$
U_{W}(g)=\left(\begin{array}{c}
I \\
w(g)
\end{array}\right)
$$

be the first factor in (3.5.3).

Definition. Let $W \in \mathcal{W}_{0}, g \in \Gamma_{+}^{W}, \kappa \in N$, then the function

$$
\psi_{W, \kappa}^{-}(g)=g U_{W}(g) \lambda^{\kappa}
$$

will be called the $\mathrm{K}$-th order Baker function of $W$.

Remark. Functions similar to (3.7.2) were introduced in [1] to study the modified KP hierarchies (see also [5]). We will give a new interpretation of these functions and we will use them to describe the KP hierarchy rather than the modified KP hierarchy. Clearly, $\psi_{w}^{-}$introduced in Section 3.5 is the 0-th order Baker function. When no confusion can arise we will frequently suppress the label 0 in this case.

As before, by $p(W)$ we denote the closed subspace $W H_{+}$. Since $W: H_{+} \mapsto$ $p(W)$ is bijective we can define the scalar product on $p(W)$ by setting

$$
(\eta, \xi)_{W}=\left\langle W^{-1} \eta, W^{-1} \xi\right\rangle,
$$

for $\eta, \xi \in p(W)$, where $\langle\cdot, \cdot\rangle$ denotes the canonical scalar product on $H_{+}$.

Lemma. Let $W \in W_{0}$ and $g \in \Gamma_{+}^{W}$. Then the collection of Baker functions $\left\{\psi_{W, K}^{\bar{N}}, \kappa \in N\right\}$ is a basis of $p(W)$ equivalent to the basis $\left\{W \lambda^{\kappa}, \kappa \in N\right\}$.

Proof. First we note that $\left\{W \lambda^{\kappa}, \kappa \in N\right\}$ is an orthonormal basis relative to the scalar product (3.7.3). Using (3.5.3) and (3.7.2) it is straightforward to show $\psi_{W, K}^{-}(g)=\left(W a^{-1} Q^{-1} W^{-1}\right) W \lambda^{k}$, whence the claim.

Corollary. For $W \in W_{0}$ and $g \in \Gamma_{+}^{W}$ define $\psi_{W, k}^{+}(g) \in H_{+}$by

$$
\psi_{W, \kappa}^{-}(g)=W \psi_{W, \kappa}^{+}(g) .
$$

Then $\left\{\psi_{W, \kappa}^{+}(g), \kappa \in N\right\}$ forms a basis of $H_{+}$equivalent to the canonical basis $\left\{\lambda^{i}\right.$, $i \geq 0\}$.

Proof. Indeed, from the proof of the lemma above we obtain $\psi_{W, K}^{+}(g)=$ $g Q^{-1}(g) \lambda^{k}$ and thus the claim.

3.8. In this section, we will prove a parallel statement to Lemma 2.5 . We parametrize $g \in \Gamma_{+}^{W}$ as before by

$$
g=e^{x \lambda+\sum_{k=2} \lambda^{\lambda} t_{k} \cdots}=e^{q(x, \lambda)} .
$$

We note that this time, as opposed to $\S 2, q$ has to satisfy certain restrictions in 
order to yield $g \in \Gamma_{+}^{W}$. In the notation of the previous section we have

Lemma. Let $W \in \mathcal{W}_{0}, g \in \Gamma_{W}^{+}$and $\kappa \geq 1$, then

$$
\psi_{W, k}^{\bar{v}}(g)=\left[\partial_{x}^{K}-\sum_{m=0}^{K-1} \partial_{x}^{m} w(g)_{-1, \kappa-m-1}\right] \psi_{W, 0}^{\bar{W}}(g),
$$

where $w(g)_{-1, j}$ is the $(-1, j)$-th matrix entry of $w(g)$ in (3.7.1).

Proof. We note first that $U_{W}(g)$ satisfies

$$
\frac{\partial}{\partial t_{k}} U_{W}(g)=-\Lambda^{k} U_{W}(g)+U_{W}(g) \Lambda_{+}^{k}+U_{W}(g) s_{k} w(g),
$$

where

$$
\Lambda^{k}=\left(\begin{array}{cc}
\Lambda_{+}^{k} & s_{k} \\
0 & \Lambda_{-}^{k}
\end{array}\right)
$$

and $t_{1}=x$. Clearly, (3.8.3) is only a restatement of the Riccati equation satisfied by $w(g)\left(=-x(-\underline{t})\right.$ in (1.5.2)). Using the definition of $\psi_{W, k}^{-}$given in (3.7.2) and the fact

$$
\frac{\partial}{\partial t_{k}} g=\Lambda^{k} g
$$

together with (3.8.3), we obtain for $j \geq 1$ and $i \geq 0$ by a straightforward computation $\frac{\partial}{\partial t_{j}} \psi_{W, \iota}(g)=\psi_{W, j+\iota}(g)+g U_{W}(g) s_{j} w(g) \lambda^{\iota}$. We note that $s_{j} w(g) \lambda^{\iota}=$ $\sum_{m=1}^{j} w(g)_{-m, \iota} \lambda^{j-m}$ holds. Thus altogether we have

$$
\frac{\partial}{\partial t_{j}} \psi_{W, \iota}(g)=\psi_{W, j+\iota}(g)+\sum_{m=1}^{j} w(g)_{-m, \iota} \psi_{W, j-m}(g) .
$$

In particular, setting $\iota=0$, we obtain

$$
\frac{\partial}{\partial t_{j}} \psi_{W, 0}^{-}(g)=\psi_{W, j}^{-}(g)+\sum_{m=1}^{i} w(g)_{-m, 0} \psi_{W, j-m}^{-}(g) .
$$

We will now explore more closely (3.8.7). For $j=1$, (3.8.7) gives

$$
\partial_{x} \psi_{W, 0}^{-}(g)=\psi_{W, 1}^{-}(g)+w(g)_{-1,0} \psi_{W, 0}^{-}(g)
$$

which in turn implies

$$
\psi_{W, 1}^{-}(g)=\left(\partial_{x}-w(g)_{-1,0}\right) \psi_{W, 0}^{-}(g)
$$

Furthermore, from (3.8.6) by choosing $j=1$ and $\iota=k-1$, we obtain

$$
\partial_{x} \psi_{W, k-1}^{\bar{N}}(g)=\psi_{W, k}^{-}(g)+w(g)_{-1, k-1} \psi_{W, 0}^{-}(g) .
$$


This can be written as

$$
\psi_{W, k}^{-}(g)=\partial_{x} \psi_{W, k-1}^{-}(g)-w(g)_{-1, k-1} \psi_{W, 0}^{-}(g) .
$$

Using the equations (3.8.9) and (3.8.11) it is straightforward to prove by induction

$$
\psi_{W, k}^{\overline{-}}(g)=\left[\partial_{x}^{k}-\sum_{m=0}^{k-1} \partial_{x}^{m} w(g)_{-1, k-m-j}\right] \psi_{W, 0}^{-}(g),
$$

whence the claim.

Remark. We would like to comment briefly on the relation of the last theorem to Sato's program of realizing infinite dimensional Grassmannians as families of $\mathscr{D}$-modulus. We refer the reader to [10; Second Lecture] for an account of his theory. Let $\mathscr{D}$ be a ring of formal differential operators, i.e.

$$
\mathscr{D}=\left\{A=\sum_{0 \leq l<x} a_{i}(x) \partial_{x}^{i} ; a_{i} \in \mathcal{O}\right\}
$$

where 0 is a differential algebra with the derivation $\partial_{1}$. We will also use the ring of formal pseudo-differential operators

$$
\mathscr{E}=\left\{A=\sum_{-x<1<\infty} a_{t}(x) \partial_{r}^{l} ; a_{i} \in \mathcal{O}\right\}
$$

In Sato's approach a central role is played by the family of left $\mathscr{D}$-submodules $\{J\}$ of $\mathscr{E}$ for which $\mathscr{E}=J \oplus \mathscr{E}^{(-1)}$, where

$$
\mathscr{E}^{(-1)}=\left\{A \in \mathscr{E} ; A=\sum_{i<0} a_{i} \partial_{x}^{i}\right\} .
$$

Any $\mathscr{D}$-submodule $J \in \mathscr{E}$ with this property is cyclic and in addition the cyclic vector $\Omega^{(0)} \in 1+\mathscr{E}(-1)$. Let $\Pi_{J}$ denote the projection on $J$ along $\mathscr{E}^{(-1)}$. Then it can be shown that the set

$$
\left\{\Omega^{(k)}=\Pi_{J}\left(\partial_{x}^{h}\right)\right\}_{k=0}^{\infty}, \partial_{x}^{0}=1,
$$

spans $J$. Moreover, the relation

$$
\partial_{x}^{k+1}=\partial_{x}\left(\partial_{x}^{k}\right)
$$

induces the relation

$$
\Omega_{k+1}-\partial_{x} \Omega_{k}+a_{k} \Omega_{0}=0
$$

where $a_{k} \in 0$.

Writing (3.8.11) as an equation for pseudo-differential operators we see that $\widehat{\psi}_{W, k}^{-}$satisfies the same equations as $\Omega_{k}$. Thus we have a direct construction of the basis $\Omega_{k}$ of the submodule $J$ out of the data $W \subset W_{0}$. Later we will show that $\widehat{\psi}_{W . k}^{-}$is actually defined on the Grassmannian $X$ (see Theorem 4.5.1). The 
method of constructing $J$ given in [10, Sect. 2.5] uses the Plücker coordinates of $W$. Subsequently $\Omega_{0}$ can be expressed in terms of Plücker coordinates. The basis of the module $J$ is then recovered from the recurrence relations given above.

3.9. The results derived in the previous sections have implications for the KP hierarchy. We recall that $\psi_{W}^{-}(g)=\psi_{W, 0}^{-}(g)$ holds.

Lemma. Let $W \in \mathcal{W}_{0}$ and $g \in \Gamma_{+}^{W}$. Then the Baker function $\psi_{W}^{-}(g)$ satisfies the KP hierarchy equations:

$$
\frac{\partial}{\partial t_{k}} \psi_{W}^{-}(g)=p_{k}\left(\partial_{x}\right) \psi_{W}^{-}(g)
$$

where $p_{k}\left(\partial_{r}\right)=\partial_{r}^{k}+\sum_{m=0}^{k-1} a_{m}(w(g)) \partial_{r}^{m}$, and $a_{m}$ are some polynomials in matrix entries of $w(g)$ and their derivatives.

Proof. Indeed, from (3.8.7) we obtain

$$
\frac{\partial}{\partial t_{k}} \psi_{W}^{-}(g)=\psi_{W, k}^{-}(g)+\sum_{m=0}^{k-1} w(g)_{m-k, 0} \psi_{W, k}^{-}(g) .
$$

Applying Lemma 3.8 to the right hand side of (3.9.2) we obtain (3.9.1).

3.10. We finish this chapter with the following generalization of [13; Proposition 5.1].

Theorem. For each $W \in \mathcal{W}_{0}$ and $g \in \Gamma_{+}^{W}$, the $k$-th order Baker function is the unique function satisfying

(i) $\psi_{W, k}^{-}(g) \in p(W)$,

(ii) $\psi_{W, k}^{-}(g)$ has the form:

$$
\psi_{W, k}^{-}(g)=g(\lambda)\left(\lambda^{k}+\sum_{i=1}^{x} a_{i}(g) \lambda^{-i}\right)
$$

\section{§4. Natural Principal Fiber Bundles}

4.1. As mentioned in the introduction we want to relate the Grassmannian manifold $X$ (see 1.2) to generalized pseudo-differential operators. The starting point for this is Theorem 2.6. To set the stage we claim the following

Lemma. ( $\left.\mathscr{W}_{0}, p, X\right)$ is a principal (Banach) fiber bundle with the structure group $G L\left(H_{+}\right)$. 
Proof. We introduce

$$
G_{e^{\prime}}=\left\{g=\left(\begin{array}{ll}
I & b \\
0 & d
\end{array}\right): g \in G\right\} .
$$

Clearly $G_{e^{\prime}}$ is a Banach subgroup of $G$. Moreover, the map $\chi: G \rightarrow \mathscr{W}_{0}$, given by

$$
\chi\left(\begin{array}{ll}
a & b \\
c & d
\end{array}\right)=\left(\begin{array}{l}
a \\
c
\end{array}\right)
$$

is onto and descends to $G / G_{e^{\prime}}$. The first fact is an easy consequence of $p$ : ${ }^{\mathcal{W}_{0}} \rightarrow$ $X$ being onto and the transitivity of the $G$ action on $X$. Moreover, $\chi$ descends to $G / G_{e^{\prime}}$ as the right multiplication by $G_{e^{\prime}}$ does not change the first column of $g \in G$. Now, let $\left(\begin{array}{ll}i & j \\ k & l\end{array}\right)$ be the inverse of $g=\left(\begin{array}{ll}a & b \\ c & d\end{array}\right)$ and $g^{\prime}$ be an element of $G$ with the same first column as $g$. Then from the equations

$$
i a+j l=I
$$

and

$$
k a+l c=0,
$$

we easily see that $g^{-1} g^{\prime} \in G_{e^{\prime}}$. Thus, if $\tilde{\chi}$ denotes the map obtained from $\chi$ by descending $\chi$ to $G / G_{e^{\prime}}$, then $\tilde{\chi}: G / G_{e^{\prime}} \rightarrow \mathcal{W}_{0}$ is bijective. Since $G / G_{e^{\prime}}$ is a Banach manifold, so is $\mathcal{W}_{0}$. We note that in (3.3.1) we had defined an action of $G \ell\left(H_{+}\right)$. on $\mathcal{W}_{0}$. It is straightforward to verify that in $G / G_{e^{\prime}}$ this action of $G \ell\left(H_{+}\right)$is given by $g G_{e^{\prime}} \rightarrow g h G_{e^{\prime}}$, where $h \in\left(\begin{array}{cc}G \ell\left(H_{+}\right) & 0 \\ 0 & I\end{array}\right)$. Next we consider the sequence

$$
G \rightarrow G / G_{e^{\prime}}=W_{0} \rightarrow G / P=X .
$$

It is easy to see that the orbits of $H$ on $\mathcal{W}_{0}$ are exactly the fibers of the canonical projection $\mathscr{W}_{0} \rightarrow X$. Using Lemma 3.3 it is not hard now to verify the claim.

4.2. It is sometimes convenient to work with another description of $\mathcal{W}_{0}$. For this we consider

$$
E=G \underset{P}{\times} G L\left(H_{+}\right)
$$

where $G=\operatorname{Aut}(X)^{0}$, the connected component of $\operatorname{Aut}(X)$ and $P=\{g \in G$; $\left.g \cdot H_{+}=H_{+}\right\}$. We recall that $G \underset{P}{\times} G L\left(H_{+}\right)=\left(G \times G L\left(H_{+}\right)\right) / \sim$, where $(g, b) \sim$ $\left(g^{\prime}, b^{\prime}\right)$ if and only if there exists $p \in P$ such that $g^{\prime}=g p$ and $b^{\prime}=\left(p^{-1}\right)_{+} b$ where for $p \in P$ we write $p=\left(\begin{array}{ll}p_{+} & * \\ 0 & *\end{array}\right)$. We will denote the equivalence class of $(g, b)$ by $\{g, b\}$. We have

Lemma. $E$ is isomorphic with $\mathcal{W}_{0}$. 
Proof. In the course of the proof of Lemma 4.1 we showed that $G / G_{e^{\prime}}$ is isomorphic with $\mathcal{W}_{0}$. Let $[g]$ be the equivalence class of $g$ in $G / G_{e^{\prime}}$. Then we define the map

$$
\mu: E \rightarrow G / G_{e^{\prime}}, \mu\{g, b\}=[g b],
$$

where we embed $G L\left(H_{+}\right)$into $G$ by $b \rightarrow\left(\begin{array}{ll}b & 0 \\ 0 & I\end{array}\right)$. Note that $\mu$ is well defined because $\mu\left\{g p,\left(p^{-1}\right)_{+} b\right\}=\left[g b p^{-1}\right]$ where $p^{\prime}=\left(p^{-1}\right)_{+} p$, and thus $\mu\{g p$, $\left.\left(p^{-1}\right)_{+} b\right\}=\mu\{g, b\}$. Moreover $\mu$ is injective. Indeed, let $\mu\{g, b\}=\mu\left\{g^{\prime}, b^{\prime}\right\}$. Thus, there exists a $q \in G_{e^{\prime}}$ such that $g^{\prime} b^{\prime}=g b q$, and we get $g^{\prime}=g p$ with $p=b q b^{\prime-1} \in P$. Moreover, $\left(p^{-1}\right)_{+}=b^{\prime} b^{-1}$ which shows that $b^{\prime}=\left(p^{-1}\right)_{+} b$. We note that $\mu$ is also onto as $\mu\{g, e\}=[g]$ for every $g \in G$. As a result, $\mu$ is bijective. Since $\mu$ is induced from the map $G \times G \ell\left(H_{+}\right) \rightarrow G,(g, b) \rightarrow g b$, the map $\mu$ is differentiable. Finally, in view of the action of $G$ it suffices to show that the tangent map of $\mu$ at $\{e, b\}$ is bijective. This is easily seen.

We finish this section by considering the canonical right action of $G L\left(H_{+}\right)$ on $E$. For $h \in G L\left(H_{+}\right)$, we set:

$$
\{g, b\} \cdot h=\{g, b h\},
$$

for all $\{g, b\} \in E$. With this action of $G L\left(H_{+}\right), \mu$ becomes a $G L\left(H_{+}\right)$-equivariant isomorphism of $\mathcal{W}_{0}$ and $E$.

4.3. In the following sections we interpret the map $\widehat{\varphi}: \mathcal{W}_{0} \rightarrow \psi$, the restriction of the map defined in Definition 2.1.2, as a bundle morphism $[4 ; \S 4$. Section 3.1]. We consider the subset $\psi\left(\Gamma_{+}\right)=\bigcup_{g \in \Gamma_{+}} \widehat{\varphi}(g) W_{0}$ of $\psi$. It is now easy to define an action of $G L\left(H_{+}\right)$on $\psi\left(\Gamma_{+}\right)$. For $a \in G \ell\left(H_{+}\right)$and $g \in \Gamma_{+}$we set

$$
\widehat{\varphi}_{W}(g) \cdot a=\widehat{\varphi}_{W a}(g) .
$$

In view of Corollary 2.5 this map is well defined. Moreover, by Theorem 2.6 we have

$$
\widehat{\varphi}_{W} \cdot a=\widehat{\varphi}_{a} \widehat{\varphi}_{W}
$$

It might appear that (4.3.2) describes a left action of $G L\left(H_{+}\right)$. That this is not so follows from the fact that $\widehat{\varphi}$ is an antihomomorphism of $G \ell\left(H_{+}\right)$(see Theorem 2.6). Moreover, since $G L\left(H_{+}\right)$acts freely on $W_{0}$ (see Lemma 3.3) Theorem 2.6 implies

Lemma. $G L\left(H_{+}\right)$acts freely on $\psi\left(\Gamma_{+}\right)$on the right.

4.4. We denote by $B\left(\Gamma_{+}\right)$the quotient space of $\psi\left(\Gamma_{+}\right)$by the equivalence relation induced by $G L\left(H_{+}\right)$. The canonical projection from $\psi\left(\Gamma_{+}\right)$to $B\left(\Gamma_{+}\right)$ 
will be denoted by $\Pi_{\psi}$. Thus we have

Theorem. $\hat{\varphi}$ is a principal isomorphism between $\left(\mathcal{W}_{0}, p, X\right)$ and $\left(\psi\left(\Gamma_{+}\right)\right.$, $\Pi_{\eta}, B\left(\Gamma_{+}\right)$), i.e. there exists a (uniquely determined) map $h: X \rightarrow B\left(\Gamma_{+}\right)$such that the following diagram is commutative

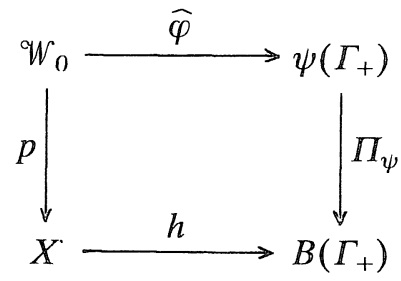

Remark. (1) The map $h$ is induced by $\widehat{\varphi}$ and therefore uniquely determined. (2) We would like to point out that we have not specified any topology on $\psi$, making the above discussion slightly formal. This deficiency keeps us from claiming that $\left(\Psi\left(\Gamma_{+}\right), \Pi_{\psi}, B\left(\Gamma_{+}\right)\right)$is locally trivial. Of course, if we give $\psi\left(\Gamma_{+}\right)$ the topology induced via $\widehat{\varphi}$, then $\left(\Psi\left(\Gamma_{+}\right), \Pi_{\psi}, B\left(\Gamma_{+}\right)\right)$becomes a principal fiber bundle with the local triviality induced from $\left(\mathcal{W}_{0}, p, X\right)$.

4.5. We have remarked at the end of Sect. 3.4 that if we take $W, W^{\prime} \in \mathcal{W}_{0}$ such that $W^{\prime}=W b$ for $b \in G L\left(H_{+}\right)$, then $\Gamma_{+}^{W}=\Gamma_{+}^{W^{\prime}}$. Thus Theorem 3.6 applies to $W$ and $W^{\prime}$ simultaneously. We claim

Theorem. Let $W, W^{\prime} \in \mathcal{W}_{0}$ and $W^{\prime}=W b$ for some $b \in G \ell\left(H_{+}\right)$. Then for all $g \in \Gamma_{+}^{W}=\Gamma_{+}^{W^{\prime}}$ we have

a)

b)

$$
\begin{aligned}
& \widehat{\psi}_{W^{\prime}}^{+}(g)=\widehat{\psi}_{W}^{+}(g) \widehat{\varphi}_{b}^{-1}(g), \\
& \widehat{\psi}_{W^{\prime}}^{-}(g)=\widehat{\psi}_{W}^{-}(g) .
\end{aligned}
$$

Proof. By Theorem 3.6 there exist unique $\widehat{\psi}_{W^{\prime}}^{+} \in \mathscr{P}_{+}, \widehat{\psi}_{W^{\prime}}^{+} \in \mathscr{P}_{-}$such that

$$
\widehat{\psi}_{W^{\prime}}^{+}(g) \widehat{\varphi}_{W^{\prime}}(g)=\widehat{\psi}_{W^{\prime}}^{-}(g), \quad g \in \Gamma_{+}^{W^{\prime}} .
$$

In view of Theorem 2.6 this gives

$$
\widehat{\psi}_{W^{\prime}}^{+}(g) \widehat{\varphi}_{b}(g) \widehat{\varphi}_{W}(g)=\widehat{\psi}_{W^{\prime}}^{-}(g), \quad g \in \Gamma_{+}^{W^{\prime}} .
$$

From the definition of $\widehat{\varphi}$ it is clear that $\widehat{\varphi}_{b}(g) \in \mathscr{P}_{+}$. Hence $\widehat{\psi}_{W^{\prime}}^{+}(g) \widehat{\varphi}_{b}(g) \in \mathscr{P}_{+}$ and $\widehat{\psi}_{W^{\prime}}^{-}(g) \in \mathscr{P}_{-}$. The uniqueness part of Theorem 3.8 applied to (2) and $W$ now yields

$$
\widehat{\psi}_{W^{\prime}}^{+}(g) \widehat{\varphi}_{b}(g)=\widehat{\psi}_{W}^{+}(g)
$$

and 


$$
\widehat{\psi}_{W^{\prime}}(g)=\widehat{\psi}_{W}^{\bar{W}}(g) .
$$

4.6. The last theorem allows one to introduce a new principal $G L\left(H_{+}\right)$bundle for which we interpret Theorem 3.6a as providing the local trivialization. First, we define

$$
\begin{aligned}
& \mathscr{F}=\bigcup_{W \in \mathcal{W}_{0}} \widehat{\varphi}_{W \mid \Gamma_{+}^{\mu}}, \\
& \mathscr{H}=\bigcup_{W \in \mathcal{W}_{0}} \widehat{\psi}_{W}^{\bar{w}} .
\end{aligned}
$$

Note that $\widehat{\psi}_{W}-$ is defined only on $\Gamma_{+}^{W}$ by (3.5.5). Next we consider the map $\Pi_{F}: \mathscr{F} \rightarrow \mathscr{H}$,

$$
\Pi_{F}\left(\widehat{\varphi}_{W}(g)\right)=\widehat{\psi}_{W}^{-}(g), \text { for } g \in \Gamma_{+}^{W} .
$$

Finally, by $r: \psi\left(\Gamma_{+}\right) \rightarrow \mathscr{F}$ we denote the restriction map:

$$
\left.\widehat{\varphi}_{W} \mapsto \widehat{\varphi}_{W}\right|_{\Gamma_{+}^{w} .}
$$

We note that the action of $G L\left(H_{+}\right)$on $\psi\left(\Gamma_{+}\right)$defines a right action of $G L\left(H_{+}\right)$ on $\mathscr{F}$. We set

$$
\left.\widehat{\varphi}_{W} \cdot a\right|_{\Gamma_{+}^{w}}=\left.\widehat{\varphi}_{W a}\right|_{\Gamma_{+}^{w_{a}}}
$$

for $a \in G L\left(H_{+}\right)$. In fact $\Gamma_{+}^{W a}=\Gamma_{+}^{W}$, so (4.6.5) is well defined. From Lemma 4.3 we get

Lemma. $G L\left(H_{+}\right)$acts freely on $\mathscr{F}$ on the right.

We summarize the present section in the following theorem.

Theorem. $\left(\mathscr{F}, \Pi_{F}, H\right)$ is a principal $G L\left(H_{+}\right)$bundle and $r$ is a principal fiber bundle isomorphism between $\left(\psi\left(\Gamma_{+}\right), \Pi_{\psi}, B\left(\Gamma_{+}\right)\right)$and $\left(\mathscr{F}_{F}, \Pi_{F}, \mathscr{H}\right)$. In particular $r$ induces a unique map $f: B\left(\Gamma_{+}\right) \rightarrow H$ making the diagram below commutative

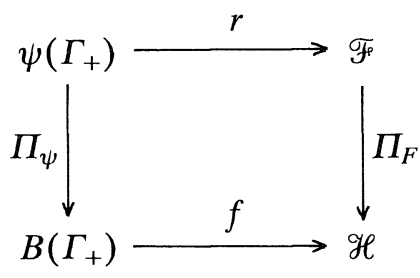


Corollary. The map $X \rightarrow H, p(W) \mapsto \widehat{\psi}_{W}^{-}$is bijective.

4.7. In this section we construct yet another principal $G L\left(H_{+}\right)$bundle which is also isomorphic to $\left(W_{0}, p, X\right)$. First we define

$$
\mathscr{F}^{\prime}=\bigcup_{W \in \mathcal{W}_{01}} \widehat{\psi}_{W}^{+}
$$

$\mathscr{F}^{\prime}$ admits a right action of $G L\left(H_{+}\right)$. Indeed, in view of Theorem 4.5, we have

$$
\widehat{\psi}_{W b}^{+}(g)=\widehat{\psi}_{W}^{+}(g) \widehat{\varphi}_{b}^{-1}(g), \quad g \in \Gamma_{+}^{W} .
$$

Thus $G L\left(H_{+}\right)$acts on $\mathscr{F}^{\prime}$ on the right.

Lemma. $G L\left(H_{+}\right)$acts freely on $\mathscr{F}^{\prime}$ on the right.

Proof. Assume that for some $W \in \mathcal{W}_{0}, b \in G L\left(H_{+}\right)$

$$
\widehat{\psi}_{W}^{+}(g) \widehat{\varphi}_{b}^{-1}(g)=\widehat{\psi}_{W}^{+}(g), \quad \text { for } g \in \Gamma_{+}^{W} .
$$

Applying both sides to $g$ we obtain $\psi_{W}^{+}(g)=\widehat{\psi}_{W}^{+}(g) g$ and $\widehat{\varphi}_{b}^{-1}(g) g=\widehat{\varphi}_{b}^{-1}(g) g=$ $b^{-1} g$, where we have used Theorem 2.6 and (2.1.6). Hence (1) implies

$$
\widehat{\psi}_{W}^{+}(g) b^{-1} g=\psi_{W}^{+}(g), \quad \text { for all } g \in \Gamma_{+}^{W} .
$$

Interchanging $b^{-1}$ and $\widehat{\psi}_{W}(g)$ we thus obtain

$$
b^{-1} \psi_{W}^{+}(g)=\psi_{W}^{+}(g), \quad \text { for all } g \in \Gamma_{+}^{W} .
$$

Next we use (3.8.2). Hence $\psi_{W, k}^{-}(g)=\mathscr{D}_{k} \psi_{W}^{-}(g)=\mathscr{D}_{k} W b^{-1} \psi_{W}^{+}(g)$, where $\mathscr{D}_{k}$ is the differential operator occurring in (3.8.2). Interchanging $\mathscr{D}_{k}$ with "constant coefficient operators" we obtain in view of (3.7.4) and the injectivity of $W$

$$
b^{-1} \psi_{W, k}(g)=\psi_{W, k}^{+}(g) \quad \text { for all } k \in N .
$$

But from Corollary 3.7 we know that the set $\left\{\psi_{W, k}^{+}(g), k \in N\right\}$ forms a basis of $H_{+}$, thus implying $b=I$.

Let $\mathscr{H}^{\prime}$ denote the quotient space of $\mathscr{F}^{\prime}$ by the equivalence relation induced by $G L\left(H_{+}\right)$. The canonical projection on $\mathscr{H}^{\prime}$ will be denoted by $\Pi_{\mathscr{F}^{\prime}}$. We have

Theorem. The map $\widehat{\psi}^{+}: \mathcal{W}_{0} \rightarrow \mathscr{F}^{\prime}, W \rightarrow \widehat{\psi}_{W}^{+} \in \mathscr{P}_{+}\left(\Gamma_{+}^{W}\right)$ is a principal isomorphism between $\left(W_{0}, p, X\right)$ and $\left(\mathscr{F}^{\prime}, \Pi_{\mathscr{F}^{\prime}}, \mathscr{H}^{\prime}\right)$. The following diagram is commutative

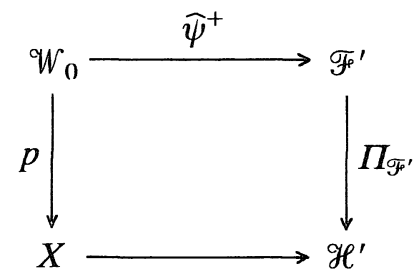




\section{§5. Differential Equations}

5.1. In this section we will discuss the action of $\Gamma_{+}$on $\mathcal{W}_{0}$ and on $\Psi\left(\Gamma_{+}\right)$as in (2.1.5).

First we define an action of $P=\left\{\left(\begin{array}{ll}a & b \\ 0 & d\end{array}\right) \in G\right\}$ on $W_{0}$. We set for $g=\left(\begin{array}{ll}a & b \\ 0 & d\end{array}\right)$ $\in P, W \in W_{0}$

$$
g \cdot W=M_{g} W a^{-1} .
$$

It is not hard to see that the projection $p: \mathcal{W}_{0} \rightarrow X$ is equivariant relative to this action of $P$. Since $\widehat{\varphi}: W_{0} \rightarrow \Psi\left(\Gamma_{+}\right)$is an isomorphism, we can transport the action of $P$ on $W_{0}$ to an action of $P$ on $\Psi\left(\Gamma_{+}\right)$such that $\widehat{\varphi}$ is $P$-equivariant, i.e.

$$
g \cdot \widehat{\varphi}_{W}=\widehat{\varphi}_{g} \cdot W, g \in P, W \in W_{0} .
$$

The action of $\Gamma_{+} \subset P$ on $\Psi\left(\Gamma_{+}\right)$takes a particularly simple form.

Lemma. Let $g \in \Gamma_{+}$and $W \in \mathcal{W}_{0}$, then

$$
\left(g \cdot \widehat{\varphi}_{W}\right)(h)=\widehat{\varphi}_{W}\left(g^{-1} h\right)
$$

Proof. From (2.1.8) and (5.1.2) we obtain $\widehat{\varphi}_{g \cdot W}(h) h=(g \cdot W) h=$ $g\left(W\left(g^{-1} h\right)\right)=g \widehat{\varphi}_{W}\left(g^{-1} h\right) g^{-1} h=g \sigma\left(\widehat{\varphi}_{W}\left(g^{-1} h\right)\right) g^{-1} h=\widehat{\varphi}_{W}\left(g^{-1} h\right) h$.

Since $\varphi_{W}(h)=\widehat{\varphi}_{W}(h) h$ it is natural to set

$$
(g \cdot \varphi)_{W}(h)=\varphi_{g \cdot W}(h), \quad g \in P, W \in \mathcal{W}_{0}, h \in \Gamma_{+} .
$$

With this notation the proof above implies

Corollary. For $g, h \in \Gamma_{+}, W \in W_{0}$ we have

$$
(g \cdot \varphi)_{W}(h)=g \varphi_{W}\left(g^{-1} h\right) .
$$

Remark. In view of Theorem 4.4 we obtain naturally an action of $P$ on $B\left(\Gamma_{+}\right)$such that the diagram of Theorem 4.4 is equivariant relative to the action of $P$ on all four occurring spaces.

5.2. In $[2 ; \S 4]$ we described all holomorphic vector fields on $X$. It was shown there that all such vector fields come from the infinitesimal action of $G$ on $X$. Clearly, (5.1.2) transports the vector fields corresponding to $\Gamma_{+}$to $\Psi\left(\Gamma_{+}\right)$. In the following sections we will describe the infinitesimal action of $\Gamma_{+}$on $\Psi\left(\Gamma_{+}\right)$ and on $\mathscr{H}$ (see 4.6).

In these discussions only subgroups of $\Gamma_{+}$are involved which are Lie subgroups of $G$. Actually, in view of the differential equations in questions we are primarily interested in the action of $\left\{e^{t_{n} \lambda^{n}}, t_{n} \in C\right\} \subset \Gamma_{+}$. 
The vector fields associated with this action will be denoted by

$$
T_{n} \widehat{\varphi}_{W}=\left.\frac{d}{d t_{n}}\right|_{t_{n}=0}\left(e^{-t_{n} \lambda^{n}} \cdot \widehat{\varphi}_{W}\right)
$$

Lemma. For $W \in W_{0}$ and $n \geq 1$ we have

$$
\begin{gathered}
T_{n} \widehat{\varphi}_{W}=-\frac{\partial}{\partial t_{n}} \widehat{\varphi}_{W} \\
\frac{\partial}{\partial t_{n}} \widehat{\varphi}_{W}(g)=\left[\partial_{x}^{n}, \widehat{\varphi}_{W}(g)\right] .
\end{gathered}
$$

Proof. Differentiating (2.1.8) for $t_{n}$ yields

$$
\frac{\partial}{\partial t_{n}} \varphi_{W}(g)=\partial_{x}^{n} \varphi_{W}(g) \text {. }
$$

Using (5.2.4) and (2.1.7) we obtain $\frac{\partial}{\partial t_{n}} \varphi_{W}(g)=\frac{\partial}{\partial t_{n}} \widehat{\varphi}_{W}(g) g=\left(\frac{\partial}{\partial t_{n}} \widehat{\varphi}_{W}(g)\right) g+$ $\widehat{\varphi}_{W}(g) \Lambda^{n} g=\partial_{x}^{n} \widehat{\varphi}_{W}(g) g$, whence (5.2.3). Next we want to compute $T_{n} \widehat{\varphi}_{W}$. In view of (5.1.3) and (5.2.1) we obtain $\left(T_{n} \widehat{\varphi}_{W}\right)(h)=\left.\frac{\partial}{\partial t_{n}}\right|_{t_{n}=0}\left(e^{t_{n} \lambda^{n}} \cdot \widehat{\varphi}_{W}\right)(h)=$ $\left.\frac{\partial}{\partial t_{n}}\right|_{t_{n}=0} \widehat{\varphi}_{W}\left(e^{-t_{n} \lambda^{n}} h\right)=-\left(\frac{\partial}{\partial t_{n}} \widehat{\varphi}_{W}\right)(h)$, whence $(5.2 .3)$.

Remark. (1) We would like to note that the equation (5.2.3) can be called the linear KP-hierarchy. Our derivation of (5.2.3) thus shows that the linear KPhierarchy appears naturally as an expression of the flow of $\Gamma_{+}$on $\Psi\left(\Gamma_{+}\right)$.

(2) The equation (5.2.4) uses substantially the fact that $\lambda^{n}$ is the product of $n$ factors $\lambda$, i.e. we use that Lie $\Gamma_{+}$is actually an associative and commutative algebra. The linear KP-hierarchy is essentially an expression of this fact.

5.3. In view of Theorem 4.6 and Section 5.1 we can induce an action of $P$ on $\mathscr{P}$ and $\mathscr{H}$ such that the diagram of Theorem 4.6 is equivariant relative to $P$.

The corresponding actions are given for $g \in P$ by

$$
\begin{gathered}
g \cdot\left(\widehat{\varphi}_{W} \mid \Gamma_{+}^{W}\right)=\widehat{\varphi}_{g \cdot W} \mid \Gamma_{+}^{g \cdot W} \\
g \cdot \widehat{\psi}_{W}^{-}=\widehat{\psi}_{g \cdot W}^{-} .
\end{gathered}
$$

As a consequence, for $g \in \Gamma_{+}, h \in \Gamma_{+}^{g \cdot W}=g^{-1} \Gamma_{+}^{W}$ we have

$$
\left(g \cdot \widehat{\psi}_{W}^{-}\right)(h)=\widehat{\psi}_{W}^{-}\left(g^{-1} h\right) \text {. }
$$

Indeed, a comparison of (5.3.2), (5.1.1) and (3.5.5) yields (5.3.3).

Consequently, defining $T_{n} \widehat{\psi}_{W}^{-}$as in (5.2.1) we obtain 


$$
T_{n} \widehat{\psi}_{W}^{-}=\frac{\partial}{\partial t_{n}} \widehat{\psi}_{W}^{-} .
$$

To find a more explicit description of $T_{n} \widehat{\varphi}_{W}$ we compare (5.3.4) to (3.9.1), where $\psi_{W}(g)=\bar{\psi}_{W} \overline{(}(g) g$ by (3.5.6). We obtain

Proposition. For the $n$-th infinitesimal flow on $\mathscr{H}$ we have

$$
T_{n} \widehat{\psi}_{W}=p_{n}\left(\partial_{x}\right) \widehat{\psi}_{W}{ }_{W}-\widehat{\psi}_{W} \partial_{x}^{n}
$$

where $p_{n}$ depends on $W$ and is given in (3.9.1).

Remark. (1) The coefficients of $p_{n}$ are differential polynomials in $u$, where $u$ is the coefficient of $\partial^{-1}$ in $\widehat{\psi}_{W}^{-}$. This follows as in the proof of [2; Proposition 6.4].

(2) Setting $K=\widehat{\psi}_{W} \overline{ }$ we have thus recaptured [13;5.8]. As a consequence, $p_{n}\left(\partial_{x}\right)=\left(K \partial_{x}^{n} K^{-1}\right)_{+}$and $Q=K \partial_{x} K^{-1}$ satisfies the Lax pair equation for the KP-hierarchy

$$
\frac{\partial}{\partial t_{n}} Q=\left[Q_{+}^{r}, Q\right]
$$

5.4. Though the Lax pair equation (5.3.6) is often used to derive the KPhierarchy we feel that the approach below may even be preferable, in particular, since it produces a hierarchy that has the usual KP-hierarchy as a consequence.

First we note that the vector fields $T_{n}$ and $T_{m}$ given by (5.3.5) commute. Thus a straightforward computation yields

Proposition. For the differential operators $p_{n}$ we have the hierarchy of equations $(n, m \geq 1)$

$$
\frac{\partial}{\partial t_{n}} p_{m}-\frac{\partial}{\partial t_{n}} p_{n}=\left[p_{n}, p_{m}\right] .
$$

As mentioned in the last section the coefficients of $p_{n}$ are differential polynomials in $u$. These polynomials can be computed inductively as explained in the proof of [2; Proposition 6.4]. However, it may be more convenient to use in place of (5.4.1) the equivalent hierarchy $[2 ; 6.3 .3]$

$$
P_{i+1} P_{j} u-P_{J+1} P_{i} u=-\sum_{k=1}^{j-1}\left(P_{k} P_{i} u\right)\left(P_{j-k} u\right)+\sum_{k=1}^{i-1}\left(P_{k} P_{j} u\right)\left(P_{i-k} u\right),
$$

where $P_{k}$ denotes the k-th Schur polynomial.

5.5. In the following sections we will discuss the invertibility of $\widehat{\psi}_{W}^{+}$and related questions. In this context we need to consider expressions like $a^{+} \circ b^{+} \circ c^{-}$where 
$a^{+}$and $b^{+}$are differential operators (of infinite order) and $c^{-}$is a pseudodifferential operator of degree 0 . We set

$$
\mathscr{D}=\left\{\sum_{n=0}^{\infty} a_{n} \partial^{n} ; a_{n}: \Gamma_{+} \rightarrow C\right\} .
$$

For a function $f=\sum_{m \in Z} f_{m}(x) \lambda^{m}$ and $a^{+} \in \mathscr{D}$ we define

$$
a^{+} f=\sum_{m \in \mathbf{Z}}\left(a^{+} f_{m}\right) \lambda^{m},
$$

wherever the expressions $a^{+} f_{m}$ make sense.

We will use frequently for $a^{+}, b^{+} \in \mathscr{D}$

$$
\left(a^{+} \circ b^{+}\right) f=a^{+}\left(b^{+} f\right) .
$$

Where the occurring changes in the order of summation are admissible, $a^{+}$。 $b^{+}$is the usual composition of two differential operators (of infinite order). This applies in particular if all $f_{m}$ and all $b_{k}$ are polynomials in $x$.

5.6. In view of (5.5.3) it is interesting to know when $\widehat{\psi}_{W}^{+}$has polynomial coefficients.

Theorem. Let $W \in \mathcal{W}_{0}$ and assume $w_{+}=I$ and that $w_{-}$has only finitely many non-zero coefficients. Then the coefficients of $\widehat{\psi}_{W}^{+}$and $\widehat{\psi}_{W}^{-}$are rational in $x$ and can be expressed as fractions with a common polynomial denominator. The coefficients of $\widehat{\varphi}_{W}$ are polynomials in $x$.

Proof. From (3.5.4) we know that the coefficients of $Q^{-1}(g) \mathbb{1}$, where $Q(g)=I+b w_{-} a^{-1}$ by (3.5.2). Writing $w_{-} \in V^{+}$in block form $\left(\begin{array}{ll}0 & q \\ 0 & 0\end{array}\right)$ and correspondingly $a^{-1}=\left(\begin{array}{ll}* & * \\ 0 & \tilde{a}^{-1}\end{array}\right)$, where $q$ and $\tilde{a}$ are $N \times N$ matrices, we see that $w_{-} a^{-1}=\left(\begin{array}{ll}0 & q \tilde{a}^{-1} \\ 0 & 0\end{array}\right)$ and $q \tilde{a}^{-1}$ is an $N \times N$-matrix the coefficients of which are polynomials in $x=t_{1}$ (and also in $t_{2}, \ldots, t_{N}$ ). Decomposing $b=\left(\begin{array}{ll}b_{1} & * \\ b_{0} & *\end{array}\right)$, we obtain

$$
Q(g)=\left(I+\left(\begin{array}{ll}
0 & b_{1} q \tilde{a}^{-1} \\
0 & b_{0} q \tilde{a}^{-1}
\end{array}\right)\right)=\left(\begin{array}{cc}
I & b_{1} q \tilde{a}^{-1} \\
0 & R
\end{array}\right)
$$

where $R=I+b_{0} q \tilde{a}^{-1}$.

Note, the coefficients of $R$ and of $b_{1} q \tilde{a}^{-1}$ are polynomials in $x=t_{1}$ (and also in $\left.t_{2}, \ldots, t_{N}\right)$. Next we consider

$$
Q(g)^{-1}=\left(\begin{array}{cc}
I & -b_{1} q \tilde{a}^{-1} R^{-1} \\
0 & R^{-1}
\end{array}\right)
$$


From this we see that the coefficients of $\sigma\left(\widehat{\psi}_{W}^{+}\right)=Q(g)^{-1} 1$ are rational functions in $x$ with the same denominator det $R$. This proves the claim for $\widehat{\psi}_{W^{\prime}}^{+}$.

Next we consider $\widehat{\varphi}_{W}$. From its definition we have

$$
\sigma\left(\widehat{\varphi}_{W}(g)\right)=M_{g}^{-1}\left(\begin{array}{l}
I \\
w_{-}
\end{array}\right) g=\left(\begin{array}{l}
Q(g) \\
d w_{-} a^{-1}
\end{array}\right) 1 .
$$

Using (1) and (3) we obtain the claim for $\widehat{\varphi}_{W}$. Finally we consider $\widehat{\psi}_{W}^{-}$. From (3.5.5) we see that $\sigma\left(\widehat{\psi}_{W}^{-}\right)=\left(\begin{array}{c}I \\ d w_{-} a^{-1} Q^{-1}(g)\end{array}\right)$. Hence the coefficients of $\widehat{\psi}_{W}^{-}$are rational with the same denominator $\operatorname{det} R$.

Remark. From (1) above it is clear that we have $\operatorname{det} R=\operatorname{det} Q(g)$. Hence the definition of $Q(g)$ shows $\tau_{W}(g)=\operatorname{det} R$. Moreover, since $d$ is upper-diagonal and $w_{-}$is a finite matrix, $\widehat{\psi}_{W}^{-}$has finitely many terms and $\widehat{\varphi}_{W}$ has finitely many terms with negative powers of $\partial_{x}$ as it can easily be seen from (3) and the formula for $\sigma\left(\widehat{\varphi}_{W}^{-}\right)$.

5.7. In this section we will address the question of invertibility of $\widehat{\psi}_{W}^{+}$. To illustrate some of the problems one encounters we will consider the simple example of $W \in W_{0}$, for which $w_{+}=I$ and

$$
w_{-1,0}=1 \text {, }
$$

while the remaining coefficients of $w_{-}$vanish. Using the notation of $\mathbf{5 . 6}$ we get

$$
R=1-x
$$

assuming that we consider only $g=e^{x \lambda}$. Thus, using (2) of the proof of Theorem 5.6 it is easy to see

$$
\widehat{\psi}_{W}^{+}=\frac{1}{1-x}\left(1-\sum_{j=1}^{\infty} \frac{(-x)^{j+1}}{(j+1) !} \partial_{x}^{j}\right)
$$

We will consider only

$$
\widehat{\xi}_{W}=1-\sum_{j=1}^{x} \frac{(-x)^{j+1}}{(j+1) !} \partial_{x}^{\prime},
$$

and ask if $\widehat{\xi}_{W}$ has a left inverse. We introduce

$$
\widehat{\zeta}=\sum_{k=0}^{\infty} \varepsilon_{k} \partial_{x}^{k}
$$

and want to determine what it means for $\widehat{\zeta}$ to satisfy

$$
\widehat{\zeta}_{\circ} \widehat{\xi}_{W}=1 \text {. }
$$

We obtain the following set of conditions 


$$
\sum_{n=0}^{r} \varepsilon_{n} \sum_{k=0}^{n}\left(\begin{array}{l}
n \\
k
\end{array}\right) \alpha_{r-k}^{(n-k)}+\sum_{n=r+1}^{\infty} \varepsilon_{n} \sum_{k=0}^{r}\left(\begin{array}{l}
n \\
k
\end{array}\right) \alpha_{r-k}^{(n-k)}=\delta_{r, 0}, \quad r \geq 0
$$

where

$$
\begin{gathered}
\alpha_{0}=1 \\
\alpha_{k}=-\frac{(-x)^{k+1}}{(k+1) !}, \quad k \geq 1 .
\end{gathered}
$$

Computing derivatives of the $\alpha_{k}$ 's with respect to $x$ we get

$$
\begin{cases}\alpha_{k}^{(m)}=(-1)^{m} \alpha_{k-m} & k>m, k \geq 1, \\ \alpha_{k}^{(k)}=(-1)^{k} x & k \geq 1, \\ \alpha_{k}^{(k+1)}=(-1)^{k} & k \geq 1 .\end{cases}
$$

After elementary computations (5.6.7) gives

$$
\begin{gathered}
\varepsilon_{0}=1 \\
\alpha_{r}+\varepsilon_{r}(1-x)-r \varepsilon_{r+1}=0, \quad r \geq 1 .
\end{gathered}
$$

Clearly, $\varepsilon_{1}$ cannot be determined from this recurrence relation. However, $\varepsilon_{r}, r>2$ is determined by $\varepsilon_{1}$. We list the first few terms

$$
\begin{gathered}
\varepsilon_{2}=\left(\alpha_{1}+\varepsilon_{1}(1-x)\right) \\
\varepsilon_{3}=\frac{1}{2}\left(\alpha_{2}+\alpha_{1}(1-x)+\varepsilon_{1}(1-x)^{2}\right.
\end{gathered}
$$

and in general we have

$$
\varepsilon_{r+1}=\sum_{m=1}^{r} \frac{(m-1) !}{r !} \alpha_{m}(1-x)^{r-m}+\frac{1}{r !} \varepsilon_{1}(1-x)^{r} .
$$

We conclude that $\widehat{\xi}_{W}$ has a family of left inverses depending on an arbitrary function $\varepsilon_{1}$. Actually we can even allow $\varepsilon_{1}$ to be a formal power series. It is not difficult to see that in this case all $\varepsilon_{n}$ are formal power series. We will see below that the equation

$$
\widehat{\xi}_{W} \circ \widehat{\zeta}_{R}=1
$$

can be solved. From (5.7.16) we obtain the following equations

$$
\begin{gathered}
\varepsilon_{0}=1, \\
\varepsilon_{1}-\frac{x^{2}}{2 !}-\sum_{n=1}^{\infty} \frac{(-x)^{n+1}}{(n+1) !} \varepsilon_{1}^{(n)}=0
\end{gathered}
$$

and 


$$
\sum_{n=0}^{r-1} \alpha_{n} \sum_{k=0}^{n}\left(\begin{array}{l}
n \\
k
\end{array}\right) \varepsilon_{r-k}^{(n-k)}+\sum_{n=r}^{\infty} \alpha_{n} \sum_{k=0}^{r}\left(\begin{array}{l}
n \\
k
\end{array}\right) \varepsilon_{r-k}^{(n-k)}=0, \quad r \geq 2 .
$$

Equation (5.7.18) has a unique formal power series solution

$$
\varepsilon_{1}=\sum_{m=2} \frac{x^{m}}{m} .
$$

If fact, if $|x|<1$, the right hand side is absolutely convergent and in this regime

$$
\varepsilon_{1}=\ln \left(\frac{1}{1-x}\right)-x \text {. }
$$

To see now that (5.7.19) can be solved we rewrite these equations in the following form:

$$
\begin{aligned}
\sum_{n=0}^{\infty} \alpha_{n}\left(\begin{array}{l}
n \\
0
\end{array}\right) \varepsilon_{r}^{(n)} & +\sum_{n=0}^{\infty} \alpha_{n+1}\left(\begin{array}{c}
n+1 \\
1
\end{array}\right) \varepsilon_{r-1}^{(n)}+\sum_{n=0}^{\infty} \alpha_{n+2}\left(\begin{array}{c}
n+2 \\
2
\end{array}\right) \varepsilon_{r-2}^{(n)} \\
& +\ldots+\sum_{n=0}^{\infty} \alpha_{n+r}\left(\begin{array}{c}
n+r \\
r
\end{array}\right) \varepsilon_{0}^{(n)}=0, \quad r \geq 2 .
\end{aligned}
$$

Because of (5.7.17) we can simplify the last term above. Thus (5.7.19) is equivalent with

(5.7.22) $\sum_{n=0} \alpha_{n} \varepsilon_{r}^{(n)}+\sum_{n=0} \alpha_{n+1}\left(\begin{array}{c}n+1 \\ 1\end{array}\right) \varepsilon_{r-1}^{(n)}+\sum_{n=0} \alpha_{n+2}\left(\begin{array}{c}n+2 \\ 2\end{array}\right) \varepsilon_{r-2}^{(n)}+\ldots+\alpha_{r}=0$

Theorem. Equation (5.7.22) has for $r \geq 1$ a unique formal power series solution such that

$$
\varepsilon_{r}=\sum_{m=r+1} \gamma_{r, m} \frac{x^{m}}{m !}
$$

and

$$
\gamma_{r, r+1}=-(r+1) ! \alpha_{r}
$$

Proof. For $r=1$ we see that (5.7.22) reduces to (5.7.18), the solution to which is (5.7.20) and thus it is of the required form. Assume that we have solved the first $r-1$ equations (5.7.27) and the solutions are of the form (5.7.23) with their first nonvanishing coefficients satisfying (5.7.24). Using $\operatorname{deg} \alpha_{0}=1$ and $\operatorname{deg} \alpha_{k}=k+1$ and expanding $\varepsilon_{r}$ into a formal power series with coefficients $\frac{\gamma_{r, m}}{m !}$ we get the following coefficients $\gamma_{r, m}$

$$
\frac{\gamma_{r, r+1}}{(r+1) !}+\alpha_{r}=0
$$




$$
\frac{\gamma_{r, m}}{m !}+\Gamma_{r, m-1}=0 \quad m>r+1
$$

where $\Gamma_{r, m-1}$ depends only on $\gamma_{r, r+1}, \ldots, \gamma_{r, m-1}$, and $\gamma_{\ell, k}$ with $\ell<r$. This shows that (5.7.22) can be solved uniquely by a formal power series, satisfying (5.7.23) and (5.7.24).

Remark. (1) We have seen that $\widehat{\xi}_{W}$ has more than one left inverse. Now we know that it also has a unique right inverse. This clearly contradicts associativity: by the nonuniqueness of the left inverse we can always choose $\widehat{\zeta}_{L} \neq \widehat{\zeta}_{R}$, e.g. by choosing $\varepsilon_{1}=0$ in $\widehat{\xi}_{L}$. Then $\widehat{\zeta}_{L} \circ\left(\widehat{\chi}_{W} \circ \widehat{\zeta}_{R}\right) \neq\left(\widehat{\zeta}_{L} \circ \widehat{\chi}_{W}\right) \circ \widehat{\zeta}_{R}$.

(2) An important consequence of this non-associativity is that the fiber bundles of generalized pseudo-differential operators cannot be interpreted in terms of "group splittings". Thus the approach of this paper differs fundamentally from the one of the paper [8]. It would be desirable to understand these different approaches from a unifying higher point of view.

(3) In spite of the fact that we have to be cautious about associativity, we obtain some "splitting" of $\widehat{\varphi}_{W}$. From Theorem 3.6 we know $\widehat{\psi}_{W}^{+}(g) \circ \widehat{\varphi}_{W}(g)=$ $\widehat{\psi}_{+}^{-}(g)$. For $W \in \mathcal{W}_{0}$ we know from Theorem 5.6 that $\widehat{\psi}_{W}^{+}(g)$ is of the form $D_{W}^{-1}(g) \widehat{\xi}_{W}(g)$. Hence $\widehat{\xi}_{W}(g) \circ \widehat{\varphi}_{W}(g)=D_{W}(g) \widehat{\psi}_{W}^{-}(g)$.

Let now $\widehat{\zeta}_{L}(g)$ denote any left-inverse of $\widehat{\zeta}_{W}(g)$ with polynomial coefficients, the existence of which has been shown in Section 5.7. Since $\widehat{\xi}_{L}, \widehat{\xi}_{W}$, and $\widehat{\varphi}_{W}$ are polynomial, we can apply (5.5.3) and obtain $\widehat{\varphi}_{W}=\widehat{\xi}_{L} \circ\left(\widehat{\xi}_{W} \circ \widehat{\varphi}_{R}\right)=\widehat{\xi}_{L} \circ D_{W} \widehat{\psi}_{W}^{-}$,

$$
\widehat{\varphi}_{W}(g)=\widehat{\zeta}_{L}(g) \circ D_{W}(g) \widehat{\psi}_{W}^{-}(g) \text {. }
$$

Therefore $\widehat{\varphi}_{W}$ splits into the product of a "differential operator" and an "integral operator". Therefore, in some sense we have obtained a "Riemann-Hilbert splitting" of $\dot{\hat{\varphi}}_{W}$.

(4) It would be very interesting to find out whether Fourier-IntegralOperators (generalizing pseudo-differential equations) can overcome the difficulties outlined above.

\section{References}

[1] Date, E., Jimbo, M.. Kashiwara, M. and Miwa, T., Transformation groups for soliton equations, Proc. RIMS Symp. Non-linear Integrable Systems. Classical Theory and Quantum Theory, World Science Publ., Singapore, 1983.

[2] Dorfmeister, J., Neher, E. and Szmigielski, J., Automorphisms of Banach manifolds associated with the KP-equation, Quart, J. Math, Oxford (2), 40 (1989), 161-195.

[3] Gohbcrg, I.C. and Krein. H.G., Introduction to the theory of linear nonselfadjoint operators, Translations of Mathematical Monographs, 18 (1969).

[4] Husemoller, D., Fiber bundles, Springer Verlag, 1975.

[5] Helminck, G.F. and Post, G.F.. Geometrical interpretation of the bilinear equations for the KP hicrarchy, Lett. in Math. Phys., 16 (1988), 359-364. 
[6] Lebedev, D.R. and Manin, Yu. I., Gel'fand-Dikii Hamiltonian operator and the coadjoint representation of the Volterra group, Funct. Anal. Appl., 13 (1979), 268-273.

[7] Manakov, S.V.. The inverse scattering transform for the time-dependent Schrödinger equation and Kadomtsev-Petviashvili cquation, Physica D, 3 (1981), 420-427.

[8] Mulase, M., Complete integrability of the Kadomtsev Petviashvili Equation, Adv. in Mathematics, 54 (1984), 57-66.

[9] Pressley, A. and Segal, G., Loop groups, Oxford, 1986.

[10] Sato, M., The KP hierarchy and infinite dimensional Grassmann Manifolds: Part I, Proceedings of Symposia in Pure Mathematics, 49 (1989).

[11] Simon, B., Trace Ideals and their applications, Cambridge University Press, Cambridge, UK. 1979.

[12] Sato, M. and Sato, Y.. Soliton equations as dynamical systems on infinite dimensional Grassmann Manifold, Lect. Notes in Num. Appl. Anal., 5 (1982), 259-271.

[13] Segal, G. and Wilson, G., Loop groups and equations of KdV type, Publ. IHES, 61 (1985).

[14] Wilson. G., Commuting flows and conscrvation laws for Lax equations, Math. Proc. Camb. Phil. Soc., 86 (1979), 131-143.

[15] Zakharov, V.E. and Shabat. A.B.. A scheme for integrating nonlinear equations of mathematical physics by the method of the inverse scattering problem. Funct. Anal. Appl., 8 (1974), $226-235$. 
